\title{
THE JOINT SIMILARITY PROBLEM FOR WEIGHTED BERGMAN SHIFTS
}

\author{
SARAH H. FERGUSON ${ }^{1}$ AND SRDJAN PETROVIC ${ }^{2}$ \\ ${ }^{1}$ Department of Mathematics, Wayne State University, Detroit, MI 48202, \\ USA (sarah@math.wayne.edu) \\ ${ }^{2}$ Department of Mathematics and Statistics, Western Michigan University, \\ Kalamazoo, MI 49008,USA (srdjan.petrovic@wmich.edu)
}

(Received 4 April 2000)

\begin{abstract}
We solve a joint similarity problem for pairs of operators of Foias-Williams/Peller type on weighted Bergman spaces. We show that for the single operator, the Hardy space theory established by Bourgain and Aleksandrov-Peller carries over to weighted Bergman spaces, by establishing the relevant weak factorizations. We then use this fact, together with a recent dilation result due to the first author and Rochberg, to show that a commuting pair of such operators is jointly polynomially bounded if and only if it is jointly completely polynomially bounded. In this case, the pair is jointly similar to a pair of contractions by Paulsen's similarity theorem.
\end{abstract}

Keywords: Bergman spaces; Hankel operators; completely bounded maps

AMS 2000 Mathematics subject classification: Primary 47B35; 47B47

\section{Introduction}

Let $A\left(\mathbb{D}^{d}\right), d \geqslant 1$, be the polydisk algebra and let $H$ be a separable Hilbert space. A unital (bounded) representation $\pi: A\left(\mathbb{D}^{d}\right) \rightarrow \mathcal{L}(H)$ is uniquely determined by the $d$ tuple of commuting operators $\left(T_{1}, \ldots, T_{d}\right), T_{i}:=\pi\left(z_{i}\right)$, where $z_{i}$ is the $i$ th coordinate function, $i=1, \ldots, d$. A necessary and sufficient condition for the boundedness of $\pi$ in terms of the commuting operators $T_{1}, \ldots, T_{d}$ is the existence of a constant $C(C=\|\pi\|)$ such that, for every polynomial $p$,

$$
\left\|p\left(T_{1}, \ldots, T_{d}\right)\right\| \leqslant C\|p\|_{\infty, \mathbb{D}^{d}}=C \sup _{z \in \mathbb{D}^{d}}\left|p\left(z_{1}, \ldots, z_{d}\right)\right| .
$$

A commuting $d$-tuple $\left(T_{1}, \ldots, T_{d}\right)$ satisfying (1.1) is said to be jointly polynomially bounded. In the case $d=1$, the operator $T_{1}$ is said to be polynomially bounded.

The map $\pi$ is completely bounded on $A\left(\mathbb{D}^{d}\right)$ if and only if the operators $T_{1}, \ldots, T_{d}$ are jointly completely polynomially bounded, which means that there is a constant $C>0$ such that, for all integers $n \geqslant 1$ and for every $n \times n$ matrix of polynomials $\left(p_{i j}\right)$,

$$
\left\|\left(p_{i j}\left(T_{1}, \ldots, T_{n}\right)\right)\right\|_{\ell_{n}^{2}(\mathcal{L}(H))} \leqslant C\left\|\left(p_{i j}\right)\right\|_{M_{n}\left(A\left(\mathbb{D}^{d}\right)\right)}=C \sup _{z \in \mathbb{D}^{d}}\left\|\left(p_{i j}(z)\right)\right\|_{M_{n}} .
$$


When $d=1$, the operator $T_{1}$ satisfying (1.2) is said to be completely polynomially bounded.

If the operators $T_{1}, \ldots, T_{d}$ are jointly polynomially bounded, then it does not necessarily follow that the $d$-tuple is jointly completely polynomially bounded, even if $d=1$. This was recently settled in [19]; [8] contains a simplified and more direct proof. The most general necessary and sufficient condition for a commuting $d$-tuple $\left(T_{1}, \ldots, T_{d}\right)$ to be jointly completely polynomially bounded is given by the following theorem, recorded below for future reference, and due to Paulsen $[\mathbf{1 5}, \mathbf{1 6}]$.

Theorem 1.1 (see $[\mathbf{1 5}, \mathbf{1 6}])$ ). A commuting $d$-tuple of operators $\left(T_{1}, \ldots, T_{d}\right)$ on a Hilbert space $H$ satisfies (1.2) if and only if there is an invertible operator $S$ on $H$ such that the commuting $d$-tuple $\left(S^{-1} T_{1} S, \ldots, S^{-1} T_{d} S\right)$ satisfies (1.2) with constant $C=1$.

We restrict our attention to the cases $d=1$ and $d=2$. Combining Theorem 1.1 with the existence of a commuting unitary dilation for a pair of commuting contractions [2], we obtain a necessary and sufficient condition for a pair of commuting operators $\left(T_{1}, T_{2}\right)$ to satisfy (1.2) for some constant $C>0$. Namely, this happens if and only if there exists an invertible operator $S$ such that $\left\|S^{-1} T_{i} S\right\| \leqslant 1, i=1,2$; in other words, if and only if the operators $T_{1}$ and $T_{2}$ are jointly similar to a pair of contractions.

The class of operators considered here are analogues of those studied in $[\mathbf{1}, \mathbf{6}-\mathbf{8}, \mathbf{1 0}, \mathbf{1 2}$, $\mathbf{1 8}, \mathbf{1 9}]$, but defined on the direct sum of two weighted Bergman spaces, instead of two copies of Hardy space. For a real number $\alpha>-1$ we define the probability measure $\mu_{\alpha}$ on $\mathbb{D}$ by

$$
\mathrm{d} \mu_{\alpha}(z)=\frac{\alpha+1}{\pi}\left(1-|z|^{2}\right)^{\alpha} \mathrm{d} A(z)
$$

where $A$ denotes the area measure on the unit disk $\mathbb{D}$. We let $T_{\alpha}$ denote multiplication by $z$ on the weighted Bergman space $L_{a}^{2}\left(\mu_{\alpha}\right)$, (the relevant definitions are contained in the next section). Let $\beta>-1$ and let $H_{b}: L_{a}^{2}\left(\mu_{\alpha}\right) \rightarrow L_{a}^{2}\left(\mu_{\beta}\right)$ be the bounded operator densely defined on polynomials by $H_{b} p=p\left(T_{\beta}^{*}\right) b$, where $b \in L_{a}^{2}\left(\mu_{\beta}\right)$. Note that $H_{b} T_{\alpha}=T_{\beta}^{*} H_{b}$. Define the $2 \times 2$ operator matrix $R_{b}$, acting on $L_{a}^{2}\left(\mu_{\alpha}\right) \oplus L_{a}^{2}\left(\mu_{\beta}\right)$, by

$$
R_{b}=\left(\begin{array}{cc}
T_{\beta}^{*} & H_{b} \\
0 & T_{\alpha}
\end{array}\right) .
$$

Our main result in $\S 4$ is Theorem 4.1 , which says that if $R_{b}$ is polynomially bounded, then it is similar to the direct sum $T_{\beta}^{*} \oplus T_{\alpha}$, and, thus, similar to a contraction. Furthermore, a necessary and sufficient condition for $R_{b}$ to be similar to a contraction is the existence of a bounded solution to the commutator equation $H_{b}=T_{\beta}^{*} X-X T_{\alpha}$, in which case the operator $X f:=H_{b} f^{\prime}$ is bounded and solves the equation.

The analogue of Theorem 4.1 for operators of the form

$$
\left(\begin{array}{cc}
S^{*} & H_{b} \\
0 & S
\end{array}\right),
$$

where $S$ is the Hardy shift and $H_{b}$ is a usual Hankel matrix, was established in the combined results of $[\mathbf{1 2}],[\mathbf{6}]$ and $[\mathbf{1}]$ and was extended to the case of shifts of finite 
multiplicity, where $H_{b}$ is now a vectorial Hankel operator, in [8]. The result does not hold for the shift of infinite multiplicity. Indeed, Pisier's example of a polynomially bounded operator which is not similar to a contraction is of the form in (1.5), where $S$ is the shift of infinite multiplicity and $H_{b} S=S^{*} H_{b}[\mathbf{1 9}]$.

The key to the solution of the similarity theorem for operators of the form (1.5) given in $[\mathbf{1}]$ is a weak factorization result for derivatives of functions in $H^{1}(\mathbb{D})$. In $\S 3$ we prove the $L_{a}^{1}\left(\mu_{\alpha}\right)$ analogue of this result, Proposition 3.2, and use the same methodology as in $[\mathbf{1}]$ and $[\mathbf{8}]$ to prove the one-variable similarity result, Theorem 4.1.

In the remainder of the paper we turn our attention to the two-variable problem by considering pairs of the form $\left(R_{b}, R_{-b}\right)$. A straightforward computation reveals that, for every polynomial $p(z, w)$,

$$
p\left(R_{b}, R_{-b}\right)=\left(\begin{array}{cc}
p\left(T_{\beta}^{*}, T_{\beta}^{*}\right) & \Delta_{b}(p) \\
0 & p\left(T_{\alpha}, T_{\alpha}\right)
\end{array}\right),
$$

where

$$
\Delta_{b}(p)=H_{b}\left(\frac{\partial p}{\partial z}-\frac{\partial p}{\partial w}\right)\left(T_{\alpha}, T_{\alpha}\right)
$$

Since $T_{\beta}$ is a contraction, von Neumann's inequality implies that the pair $\left(T_{\beta}^{*}, T_{\beta}^{*}\right)$ satisfies (1.2) with constant $C=1$. The same result (for the same reason) holds for the pair $\left(T_{\alpha}, T_{\alpha}\right)$. It follows that the pair $\left(R_{b}, R_{-b}\right)$ is jointly (completely) polynomially bounded if and only if $\Delta_{b}$ extends to a (completely) bounded map of the bidisk algebra, $A\left(\mathbb{D}^{2}\right)$, into $\mathcal{L}\left(L_{a}^{2}\left(\mu_{\alpha}\right), L_{a}^{2}\left(\mu_{\beta}\right)\right)$; in other words, if and only if there is a constant $C>0$ such that for all integers $n \geqslant 1$ and for all $n \times n$ matrices of polynomials $\left(p_{i j}\right)$,

$$
\left\|\left(\Delta_{b}\left(p_{i j}\right)\right)\right\|_{L_{a}^{2}\left(\mu_{\alpha}\right) \rightarrow L_{a}^{2}\left(\mu_{\beta}\right)} \leqslant C\left\|\left(p_{i j}\right)\right\|_{M_{n}\left(A\left(\mathbb{D}^{2}\right)\right)}=C \sup _{z \in \mathbb{D}^{2}}\left\|\left(p_{i j}(z)\right)\right\|_{M_{n}} .
$$

We show in Theorem 5.4 that if $\Delta_{b}$ is bounded on $A\left(\mathbb{D}^{2}\right)$, then it is completely bounded. We do this by realizing $\Delta_{b}$ as a pushout of a completely bounded derivation $\delta^{\gamma}, \gamma=\frac{1}{2} \alpha+1$. In other words, we will show that if $\Delta_{b}$ is bounded on $A\left(\mathbb{D}^{2}\right)$, then there is a bounded operator $A: L_{a}^{2}\left(\mu_{\alpha+2}\right) \rightarrow L_{a}^{2}\left(\mu_{\beta}\right)$ satisfying $A T_{\alpha+2}=T_{\beta}^{*} A$, (i.e. $A$ is a weighted Hankel operator) and such that $\Delta_{b}(\cdot)=A \delta^{\gamma}(\cdot)$. The construction is based on a dilation result from [11] and the fact that, for a polynomial $p(z, w), \delta^{\gamma}(p)$ is just a part of $p\left(M_{z_{1}}, M_{z_{2}}\right)$. Here $M_{z_{i}}$ is multiplication by the $i$ th coordinate function $z_{i}, i=1,2$, acting on the Hilbert space generated by the kernel $k_{\gamma}$, defined on $\mathbb{D}^{2} \times \mathbb{D}^{2}$ by

$$
k_{\gamma}(z, w)=\frac{1}{\left(1-z_{1} \overline{w_{1}}\right)^{\gamma}\left(1-z_{2} \overline{w_{2}}\right)^{\gamma}} .
$$

For $\gamma \geqslant 1$ (i.e. $\alpha \geqslant 0$ ) the map $p \mapsto p\left(M_{z_{1}}, M_{z_{2}}\right)$ is completely contractive on $A\left(\mathbb{D}^{2}\right)$ but, for $0<\gamma<1$ (i.e. $-1<\alpha<0$ ), the operator $M_{z_{1}}$ is not even power bounded and, hence, the pair $\left(M_{z_{1}}, M_{z_{2}}\right)$ is not jointly polynomially bounded. Nevertheless, we do show in Theorem 5.3 that $\delta^{\gamma}$ is completely bounded on $A\left(\mathbb{D}^{2}\right)$ for all $\gamma>0$ (i.e. for all $\alpha>-1$ ), by exploiting a well-known characterization of the Bloch class in terms of Carleson measures on Bergman spaces given in Proposition 2.1. 
As a corollary to Theorem 5.4 we deduce that the pair $\left(R_{b}, R_{-b}\right)$ is jointly polynomially bounded if and only if it is jointly completely polynomially bounded and thus, by Theorem 1.1 and Ando's dilation theorem, if and only if the operators $R_{b}$ and $R_{-b}$ are jointly similar to a pair of contractions. Furthermore, a necessary and sufficient condition for the pair to satisfy the estimate in (1.1) is that the single operator $R_{b}$ is polynomially bounded, Corollary 5.5. It is still an open problem if the analogue of Theorem 5.4 (or Corollary 5.5) holds for a pair of operators of the form in (1.5). This problem was brought to our attention by Paulsen.

It appears that the situation in the Hardy space may be quite different. Pisier has constructed, in [20], a pair of operators (using the shift of infinite multiplicity) each of which is similar to a contraction (hence polynomially bounded), but the pair is not jointly polynomially bounded. These operators do not have Hankelian entries in the $(1,2)$ corner, nor is the difference between the $(1,2)$ corners a Hankel operator. For this reason, Pisier's example falls outside the scope of our framework, but this leads to an interesting open question, especially in view of Corollary 5.8. Namely, what can be said about Pisier's pair in the context of (weighted) Bergman spaces?

The paper is organized as follows. Section 2 contains the relevant facts on weighted Bergman spaces and Bloch functions that will be used throughout the paper. The results in $\S 2$ are well known (the book [29] is a good reference). Section 3 contains weak factorization results for weighted Bergman spaces and boundedness criteria for weighted Hankel operators between Bergman spaces. Good references for material on Hankel forms between weighted Bergman spaces include $[\mathbf{3}, \mathbf{4}, \mathbf{1 3}, \mathbf{1 4}, \mathbf{1 7}, \mathbf{2 1}, \mathbf{2 2}, \mathbf{2 6}, \mathbf{2 8}]$. Section 4 contains the proof of the one-variable similarity result and $\S 5$ is devoted to the two-variable case. We also include in $\S 5$ the relevant details from the dilation result in [11] that is fundamental in the proof of the joint similarity result.

\section{Preliminaries on weighted Bergman spaces}

The weighted Bergman space (on the unit disk) corresponding to the weight $\mu_{\alpha}$ will be denoted by $L_{a}^{p}\left(\mu_{\alpha}\right), p \geqslant 1$. We write $L_{a}^{p}$ for the unweighted space, i.e. when $\alpha=0$. By definition, $L_{a}^{p}\left(\mu_{\alpha}\right)$ is the closure of the analytic polynomials in $L^{p}\left(\mathbb{D}, \mu_{\alpha}\right)$ and coincides with the class of holomorphic functions on the unit disk which are $p$-integrable with respect to the measure $\mu_{\alpha}$.

The space $L_{a}^{2}\left(\mu_{\alpha}\right)$ is a reproducing kernel Hilbert space with reproducing kernel

$$
K_{\alpha}(\lambda, z)=\frac{1}{(1-\bar{z} \lambda)^{\alpha+2}} .
$$

In particular, we can represent any function $f \in L_{a}^{1}\left(\mu_{\alpha}\right)$ at the point $z \in \mathbb{D}$ by the integral

$$
f(z)=\int_{\mathbb{D}} f(\lambda) \overline{K_{\alpha}(\lambda, z)} \mathrm{d} \mu_{\alpha}(\lambda) .
$$

An immediate consequence of this formula is that for each $z \in \mathbb{D}$, evaluation at $z$ is a bounded linear functional on $L_{a}^{p}\left(\mu_{\alpha}\right)$ for all $p \geqslant 1$. 
The Taylor expansion of $K_{\alpha}(\cdot, z)$ about $\lambda=0$ yields

$$
K_{\alpha}(\lambda, z)=\sum_{n=0}^{\infty} \frac{(\alpha+2)_{n}}{n !} \lambda^{n} \bar{z}^{n}
$$

where, for $\gamma>0$ and $n \geqslant 0$,

$$
(\gamma)_{n}:=\frac{\Gamma(n+\gamma)}{\Gamma(\gamma)}= \begin{cases}1, & n=0 \\ \gamma(\gamma+1) \ldots(\gamma+n-1), & n \geqslant 1\end{cases}
$$

Thus, $L_{a}^{2}\left(\mu_{\alpha}\right)$ is the weighted Hardy space with orthonormal basis

$$
e_{n, \alpha}(z)=\sqrt{\frac{(\alpha+2)_{n}}{n !}} z^{n}, \quad n \geqslant 0 .
$$

By Stirling's formula, $n ! /(\alpha+2)_{n} \sim 1 /(n+1)^{\alpha+1}$ and so, for any polynomial $p(z)=$ $\sum_{n=0}^{m} a_{n} z^{n}$,

$$
\|p\|_{L_{a}^{2}\left(\mu_{\alpha}\right)}^{2}=\sum_{n=0}^{m} \frac{\left|a_{n}\right|^{2} n !}{(\alpha+2)_{n}} \sim \sum_{n=0}^{m} \frac{\left|a_{n}\right|^{2}}{(n+1)^{1+\alpha}} .
$$

Using the orthonormal basis of $L_{a}^{2}\left(\mu_{\alpha}\right)$ given in (2.4), we see that the operator $M_{z}$ of multiplication by the coordinate function $z$ on $L_{a}^{2}\left(\mu_{\alpha}\right)$ is unitarily equivalent to a contractive weighted shift on $\ell^{2}$ with weight sequence

$$
w_{n}=\sqrt{\frac{n+1}{n+\alpha+2}}, \quad n \geqslant 0 .
$$

The Bloch space, denoted by BLOCH, is defined to be the space of analytic functions on $\mathbb{D}$ such that

$$
\|b\|_{*}=\sup _{z \in \mathbb{D}}\left|b^{\prime}(z)\right|\left(1-|z|^{2}\right)<+\infty .
$$

The norm $\|b\|_{\mathrm{BLOCH}}=|b(0)|+\|b\|_{*}$ is a complete norm on BLOCH and, under the usual (conjugate linear) integral pairing

$$
\langle f, b\rangle=\int_{\mathbb{D}} f(z) \overline{b(z)} \mathrm{d} \mu_{\alpha}(z),
$$

the space BLOCH can be identified with the Banach space dual of $L_{a}^{1}\left(\mu_{\alpha}\right)$ for all $\alpha>-1$. This fact is well known and easily follows from Lemma 2.5 below and Hölder's inequality.

An alternative characterization of the Bloch class can be given in terms of the finiteness of a certain multiplier norm. For a holomorphic function $b$ let $M_{b}$ denote the operator of pointwise multiplication by $b$. Then, for $\alpha, \beta>-1$,

$$
\left\|M_{b}\right\|_{L_{a}^{2}\left(\mu_{\alpha}\right) \rightarrow L_{a}^{2}\left(\mu_{\beta}\right)}=\sup _{\|f\|_{L_{a}^{2}\left(\mu_{\alpha}\right)}=1}\|b f\|_{L_{a}^{2}\left(\mu_{\beta}\right)} .
$$

The equivalence of the semi-norms, $\left\|M_{b^{\prime}}\right\|$ and $\|b\|_{*}$, is well known [25], but we will include a proof of the following estimate, which will be used in $\S 5$. 
Proposition 2.1. Fix $\alpha>-1$ and let $b$ be holomorphic on $\mathbb{D}$. Then

$$
\|b\|_{*} \leqslant\left\|M_{b^{\prime}}\right\|_{L_{a}^{2}\left(\mu_{\alpha}\right) \rightarrow L_{a}^{2}\left(\mu_{\alpha+2}\right)} \leqslant \sqrt{\frac{\alpha+3}{\alpha+1}}\|b\|_{*} .
$$

Proof. Let $z, w \in \mathbb{D}$. Clearly,

$$
\left\langle M_{b^{\prime}}^{*} K_{\alpha+2}(\cdot, w), K_{\alpha}(\cdot, z)\right\rangle_{L_{a}^{2}\left(\mu_{\alpha}\right)}=\left\langle K_{\alpha+2}(\cdot, w), M_{b^{\prime}} K_{\alpha}(\cdot, z)\right\rangle_{L_{a}^{2}\left(\mu_{\alpha+2}\right)},
$$

and the reproducing properties of $K_{\alpha}$ and $K_{\alpha+2}$ show that

$$
M_{b^{\prime}}^{*} K_{\alpha+2}(z, w)=\overline{b^{\prime}(w)} K_{\alpha}(z, w) .
$$

It follows easily from (2.6) that

$$
\left\langle M_{b^{\prime}}^{*} K_{\alpha+2}(\cdot, z), M_{b^{\prime}}^{*} K_{\alpha+2}(\cdot, w)\right\rangle_{L_{a}^{2}\left(\mu_{\alpha}\right)}=\overline{b^{\prime}(z)} b^{\prime}(w) K_{\alpha}(w, z) .
$$

In particular, when $w=z$, we obtain that

$$
\begin{aligned}
\left|b^{\prime}(z)\right|^{2} K_{\alpha}(z, z) & =\left\|M_{b^{\prime}}^{*} K_{\alpha+2}(\cdot, z)\right\|_{L_{a}^{2}\left(\mu_{\alpha}\right)}^{2} \\
& \leqslant\left\|M_{b^{\prime}}^{*}\right\|_{L_{a}^{2}\left(\mu_{\alpha+2}\right) \rightarrow L_{a}^{2}\left(\mu_{\alpha}\right)}^{2}\left\|K_{\alpha+2}(\cdot, z)\right\|_{L_{a}^{2}\left(\mu_{\alpha}\right)}^{2} \\
& =\left\|M_{b^{\prime}}\right\|_{L_{a}^{2}\left(\mu_{\alpha}\right) \rightarrow L_{a}^{2}\left(\mu_{\alpha+2}\right)}^{2} K_{\alpha+2}(z, z) .
\end{aligned}
$$

This implies that $\|b\|_{*} \leqslant\left\|M_{b^{\prime}}\right\|_{L_{a}^{2}\left(\mu_{\alpha}\right) \rightarrow L_{a}^{2}\left(\mu_{\alpha+2}\right)}$.

On the other hand, if $f \in L_{a}\left(\mu_{\alpha}\right),\|f\|_{L_{a}\left(\mu_{\alpha}\right)}^{2}=1$, then, by Hölder's inequality,

$$
\begin{aligned}
\left\|b^{\prime} f\right\|_{L_{a}^{2}\left(\mu_{\alpha+2}\right)}^{2} & =(\alpha+3) \int_{\mathbb{D}}\left|b^{\prime}(z)\right|^{2}\left(1-|z|^{2}\right)^{2}|f(z)|^{2}\left(1-|z|^{2}\right)^{\alpha} \mathrm{d} A(z) \\
& \leqslant\left(\frac{\alpha+3}{\alpha+1}\right)\|b\|_{*}^{2} .
\end{aligned}
$$

For any positive integer $k$, let $D^{k}$ denote the $k$ th-order derivative operator, i.e. $D^{k} f=$ $f^{(k)}$. When $k=1$ we write $D$ instead of $D^{1}$. If $\mathcal{X}$ is a Banach space of holomorphic functions on some domain, then the space of $k$ th-order derivatives of functions in $\mathcal{X}$ will be denoted by $D^{k} \mathcal{X}$. In other words,

$$
D^{k} \mathcal{X}=\left\{f^{(k)} \mid f \in \mathcal{X}\right\}
$$

The space $D^{k} \mathcal{X}$ will be viewed as a Banach space with the natural quotient norm $\|g\|=$ $\inf \|f\|_{\mathcal{X}}$, the infimum being over all functions $f \in \mathcal{X}$ with $g=f^{(k)}$. The next result is just a reformulation of Theorem 4.2.9 in $[\mathbf{2 9}]$ in terms of the spaces $D^{k} L_{a}^{p}$, and so the proof will be omitted here.

Proposition 2.2. $D^{k} L_{a}^{p}=L_{a}^{p}\left(\mu_{p k}\right)$ with equivalent norms, for all $p \geqslant 1$ and all integers $k \geqslant 0$. 
Using the characterization of the norm on $L_{a}^{2}\left(\mu_{\alpha}\right)$ given in (2.5), we see that a function $f$ belongs to $L_{a}^{2}\left(\mu_{\alpha}\right)$ if and only if $f^{\prime} \in L_{a}^{2}\left(\mu_{\alpha+2}\right)$. Thus, $D L_{a}^{2}\left(\mu_{\alpha}\right)=L_{a}^{2}\left(\mu_{\alpha+2}\right)$ and, furthermore, the norms are comparable for all $\alpha>-1$. This fact has an $L^{1}$ analogue (Proposition 2.4). Before proving this we make the following observation.

Lemma 2.3. $\left\langle f^{\prime}, g\right\rangle_{L_{\alpha}^{2}\left(\mu_{\alpha+1}\right)}=(\alpha+2)\left\langle f, M_{z} g\right\rangle_{L_{\alpha}^{2}\left(\mu_{\alpha}\right)}$ for all $\alpha>-1$.

Proof. Let

$$
f(z)=\sum_{n=0}^{N} a_{n} z^{n} \quad \text { and } \quad g(z)=\sum_{m=0}^{M} b_{m} z^{m} .
$$

By (2.4),

$$
\int_{\mathbb{D}} z^{j} \overline{z^{j}} \mathrm{~d} \mu_{\alpha+1}(z)=\frac{j !}{(\alpha+3)_{j}} .
$$

Since $(\alpha+2)_{j+1}=(\alpha+2)(\alpha+3)_{j}$, we have that

$$
\begin{aligned}
\left\langle f^{\prime}, g\right\rangle_{L_{a}^{2}\left(\mu_{\alpha+1}\right)} & =\sum_{j=0}^{\infty}(j+1) a_{j+1} \overline{b_{j}} \frac{j !}{(\alpha+3)_{j}} \\
& =(\alpha+2) \sum_{j=0}^{\infty} a_{j+1} \overline{b_{j}} \frac{(j+1) !}{(\alpha+2)_{j+1}} \\
& =(\alpha+2)\left\langle f, M_{z} g\right\rangle_{L_{a}^{2}\left(\mu_{\alpha}\right) .}
\end{aligned}
$$

Proposition 2.4. $D L_{a}^{p}\left(\mu_{\alpha}\right)=L_{a}^{p}\left(\mu_{\alpha+p}\right)$ with equivalent norms, for all $\alpha>-1$ and $p=1,2$.

Proof. Let $b \in \mathrm{BLOCH}$ and $f \in L_{a}^{1}\left(\mu_{\alpha}\right), f(0)=0$. Since $L_{a}^{1}\left(\mu_{\beta}\right)^{*}=\mathrm{BLOCH}$ for all $\beta \geqslant 0$, we have by Lemma 2.3 that

$$
\left\|f^{\prime}\right\|_{L_{a}^{1}\left(\mu_{\alpha+1}\right)} \sim \sup _{\|b\|_{\text {BLOCH }}=1}\left|\int_{\mathbb{D}} \bar{b} f^{\prime} \mathrm{d} \mu_{\alpha+1}\right| \sim \sup _{\|b\|_{\text {BLOCH }}}\left|\int_{\mathbb{D}} \overline{z b} f \mathrm{~d} \mu_{\alpha}\right| .
$$

Thus, it remains to show that $\|b\|_{\mathrm{BLOCH}}$ and $\|z b\|_{\mathrm{BLOCH}}$ are comparable. By duality we see that

$$
\|z b\|_{\mathrm{BLOCH}} \sim \sup _{\|g\|_{L_{a}^{1}}=1}\left|\int_{\mathbb{D}} \overline{z b} g \mathrm{~d} A\right|=\sup _{\substack{\|g\|_{L_{a}^{1}=1} \\ g(0)=0}}\left|\int_{\mathbb{D}} \overline{z b} g \mathrm{~d} A\right|=\sup _{\substack{\|g\|_{L_{a}^{1}=1} \\ g(0)=0}}\left|\int_{\mathbb{D}} \bar{b} g^{\prime} \mathrm{d} \mu_{1}\right| .
$$

By Proposition 2.2, $L_{a}^{1}\left(\mu_{1}\right)=D L_{a}^{1}$ with comparable norms, which implies that the supremum above on the right is equivalent to $\|b\|_{\mathrm{BLOCH}}$. This completes the proof of the case $p=1$. As mentioned above, the case $p=2$ follows from (2.5).

We end this section with two computational lemmas that will be useful later.

Lemma 2.5. Let $\beta \geqslant \alpha>-1$. The following are equivalent for a function $f \in L_{a}^{1}$. 
(1) There is a constant $C>0$ such that $\left|\langle f, g\rangle_{L_{a}^{2}\left(\mu_{\beta}\right)}\right| \leqslant C\|g\|_{L_{a}^{1}\left(\mu_{\alpha}\right)}$, for all functions $g$ in $L_{a}^{1}\left(\mu_{\alpha}\right)$.

(2) There is a constant $C>0$ such that $\left|f^{\prime}(z)\right|\left(1-|z|^{2}\right)^{\beta-\alpha+1} \leqslant C$, for all $z \in \mathbb{D}$.

Proof. By (2.2),

$$
f(z)=\int_{\mathbb{D}} \frac{f(\lambda)}{(1-\bar{\lambda} z)^{\beta+2}} \mathrm{~d} \mu_{\beta}(\lambda) .
$$

Differentiating under the integral sign gives

$$
f^{\prime}(z)=(\beta+2) \int_{\mathbb{D}} \frac{f(\lambda) \bar{\lambda}}{(1-\bar{\lambda} z)^{\beta+3}} \mathrm{~d} \mu_{\beta}(\lambda)
$$

If (1) holds, then

$$
\left|f^{\prime}(z)\right| \leqslant C(\beta+2)\left\|\frac{1}{(1-\bar{\lambda} z)^{\beta+3}}\right\|_{L_{a}^{1}\left(\mu_{\alpha}\right)}, \quad \forall z \in \mathbb{D} .
$$

Since $\beta+3=\alpha+2+(\beta-\alpha+1)$, we have that

$$
\left\|\frac{1}{(1-\bar{\lambda} z)^{\beta+3}}\right\|_{L_{a}^{1}\left(\mu_{\alpha}\right)} \sim \frac{1}{\left(1-|z|^{2}\right)^{\beta-\alpha+1}},
$$

(see [29, Lemma 4.2.2]). The estimate in (2) now follows.

Suppose now that (2) holds. Then, by Lemma 2.3 and Hölder's inequality,

$$
(\beta+2)\left|\left\langle f, M_{z} g\right\rangle_{L_{a}^{2}\left(\mu_{\beta}\right)}\right|=\left|\left\langle f^{\prime}, g\right\rangle_{L_{a}^{2}\left(\mu_{\beta+1}\right)}\right| \leqslant C\|g\|_{L_{a}^{1}\left(\mu_{\alpha}\right)} .
$$

Notice that $z L_{a}^{1}\left(\mu_{\alpha}\right)=\left\{f \in L_{a}^{1}\left(\mu_{\alpha}\right) \mid f(0)=0\right\}$. In particular, $z L_{a}^{1}\left(\mu_{\alpha}\right)$ is closed in $L_{a}^{1}\left(\mu_{\alpha}\right)$. Thus, since $M_{z}$ is injective, $\|g\|_{L_{a}^{1}\left(\mu_{\alpha}\right)} \leqslant C^{\prime}\left\|M_{z} g\right\|_{L_{a}^{1}\left(\mu_{\alpha}\right)}$ for some $C^{\prime}>0$. It follows, by the inequality above, that

$$
\left|\langle f, g\rangle_{L_{a}^{2}\left(\mu_{\beta}\right)}\right| \leqslant \tilde{C}\|g\|_{L_{a}^{1}\left(\mu_{\alpha}\right)}
$$

for some constant $\tilde{C}>0$ and all functions $g$ in $L_{a}^{1}\left(\mu_{\alpha}\right)$ with $g(0)=0$. The general case asserted in (1) can now be obtained by writing $g(z)=g(0)+g_{1}(z)$, and using the fact that evaluation at $z=0$ is bounded on $L_{a}^{1}\left(\mu_{\alpha}\right)$.

Lemma 2.6. Let $\beta \geqslant \alpha>-1$ and let $f \in L_{a}^{1}$. Then

$$
\sup _{\|g\|_{L_{a}^{1}\left(\mu_{\alpha}\right)}=1}\left|\left\langle f, g^{\prime}\right\rangle_{L_{a}^{2}\left(\mu_{\beta}\right)}\right|<\infty \Leftrightarrow \sup _{\|g\|_{L_{a}^{1}\left(\mu_{\alpha}\right)}=1}\left|\left\langle f^{\prime}, g\right\rangle_{L_{a}^{2}\left(\mu_{\beta}\right)}\right|<\infty .
$$

Proof. It is easy to see, using Lemma 2.5 , that the supremum above on the right is finite if and only if there is a constant $\tilde{C}>0$ such that

$$
\left|\left\langle f^{\prime}, g\right\rangle_{L_{a}^{2}\left(\mu_{\beta+1}\right)}\right| \leqslant \tilde{C}\|g\|_{L_{a}^{1}\left(\mu_{\alpha+1}\right)}, \quad \forall g \in L_{a}^{1}\left(\mu_{\alpha+1}\right) .
$$


By Lemma 2.3, $\left\langle f^{\prime}, g\right\rangle_{L_{a}^{2}\left(\mu_{\beta+1}\right)}=(\beta+2)\left\langle f, M_{z} g\right\rangle_{L_{a}^{2}\left(\mu_{\beta}\right)}$ and thus, inequality (2.7) becomes

$$
\left|\left\langle f, M_{z} g\right\rangle_{L_{a}^{2}\left(\mu_{\beta}\right)}\right| \leqslant \frac{\tilde{C}}{\beta+2}\|g\|_{L_{a}^{1}\left(\mu_{\alpha+1}\right)}, \quad \forall g \in L_{a}^{1}\left(\mu_{\alpha+1}\right) .
$$

Since $M_{z}$ is bounded below on $L_{a}^{1}\left(\mu_{\alpha+1}\right)$, inequality (2.8) is equivalent to

$$
\left|\langle f, g\rangle_{L_{a}^{2}\left(\mu_{\beta}\right)}\right| \leqslant C_{1}\|g\|_{L_{a}^{1}\left(\mu_{\alpha+1}\right)}, \quad \forall g \in L_{a}^{1}\left(\mu_{\alpha+1}\right), \quad g(0)=0 .
$$

Using the same argument as in the proof of Lemma 2.5 we see that (2.9) holds if and only if the corresponding inequality is true for all $g \in L_{a}^{1}\left(\mu_{\alpha+1}\right)$. Finally, by Proposition 2.4, $D L_{a}^{1}\left(\mu_{\alpha}\right)=L_{a}^{1}\left(\mu_{\alpha+1}\right)$ with comparable norms and so inequality (2.9) is equivalent to the condition that the supremum above on the left is finite.

\section{Weak factorization and weighted Hankel operators}

Let $\mathcal{X}$ and $\mathcal{Y}$ be Banach spaces of holomorphic functions on some domain. If $\left(f_{i}\right) \subseteq \mathcal{X}$, $\left(g_{i}\right) \subseteq \mathcal{Y}$, and $h=\sum_{i=0}^{n} f_{i} g_{i}$, we define the norm

$$
\|h\|=\inf _{h=\sum_{i=1}^{n} f_{i} g_{i}} \sum_{i=0}^{n}\left\|f_{i}\right\|_{\mathcal{X}}\left\|g_{i}\right\|_{\mathcal{Y}} .
$$

The space $\mathcal{X} \odot \mathcal{Y}$ is defined to be the completion of the linear span of functions of the form $f g$, with $f \in \mathcal{X}, g \in \mathcal{Y}$, with respect to the norm (3.1). The space $\mathcal{X} \odot \mathcal{Y}$ is a Banach space which is isomorphic to a quotient of the projective tensor product of $\mathcal{X}$ and $\mathcal{Y}$. In this section we will establish several assertions of the form $\mathcal{Z}=\mathcal{X} \odot \mathcal{Y}$. Such an equality is often referred to as a weak factorization of the space $\mathcal{Z}$. In our work we will omit the word weak and speak just of a factorization.

The factorization below is well known (cf. [27]), but we include a proof for the sake of completeness.

Proposition 3.1. Let $\alpha, \beta>-1$. Then $L_{a}^{2}\left(\mu_{\alpha}\right) \odot L_{a}^{2}\left(\mu_{\beta}\right)=L_{a}^{1}\left(\mu_{(\alpha+\beta) / 2}\right)$ with comparable norms.

Proof. Hölder's inequality shows that there is a contractive inclusion

$$
j: L_{a}^{2}\left(\mu_{\alpha}\right) \odot L_{a}^{2}\left(\mu_{\beta}\right) \hookrightarrow L_{a}^{1}\left(\mu_{(\alpha+\beta) / 2}\right) .
$$

To show that $j$ is surjective it suffices to show that its adjoint $j^{*}$ is bounded below. Identifying the dual of $L_{a}^{1}\left(\mu_{(\alpha+\beta) / 2}\right)$ with $\mathrm{BLOCH}$, we see that $j^{*}$ is bounded below if and only if there is $C>0$ such that

$$
\|b\|_{\mathrm{BLOCH}} \leqslant C \sup \left\{\left|\int_{\mathbb{D}} \bar{b} f g \mathrm{~d} \mu_{(\alpha+\beta) / 2}\right|:\|f\|_{L_{a}^{2}\left(\mu_{\alpha}\right)}=1,\|g\|_{L_{a}^{2}\left(\mu_{\beta}\right)}=1\right\} .
$$

Suppose that the supremum above is finite and denote this number by $M$. By (2.2),

$$
b(z)=\int_{\mathbb{D}} b(\lambda) \frac{1}{(1-\bar{\lambda} z)^{((\alpha+\beta) / 2)+2}} \mathrm{~d} \mu_{(\alpha+\beta) / 2}(\lambda) .
$$


Differentiating under the integral sign gives

$$
b^{\prime}(z)=\left(\frac{1}{2}(\alpha+\beta)+2\right) \int_{\mathbb{D}} b(\lambda) \frac{\bar{\lambda}}{(1-\bar{\lambda} z)^{((\alpha+\beta) / 2)+3}} \mathrm{~d} \mu_{(\alpha+\beta) / 2}(\lambda) .
$$

Hence, by definition of $M$,

$$
\left|b^{\prime}(z)\right| \leqslant M\left(\frac{1}{2}(\alpha+\beta)+2\right)\left\|\frac{1}{(1-\bar{\lambda} z)^{(\alpha+3) / 2}}\right\|_{L_{a}^{2}\left(\mu_{\alpha}\right)}\left\|\frac{1}{(1-\bar{\lambda} z)^{(\beta+3) / 2}}\right\|_{L_{a}^{2}\left(\mu_{\beta}\right)} .
$$

One knows that both of the norms above are comparable with $\left(1-|z|^{2}\right)^{-1 / 2}$ on $\mathbb{D}$ (cf. $[\mathbf{2 9}$, Lemma 4.2.2]), and so it follows that there is a constant $C>0$ such that $\left|b^{\prime}(z)\right| \leqslant$ $M C /\left(1-|z|^{2}\right)$ for all $z \in \mathbb{D}$. Thus (3.2) holds and the proposition is proved.

The next factorization is the $L_{a}^{1}$ analogue of the Hardy space result $D A(\mathbb{D}) \odot H^{p}=$ $D H^{p}$, for $p \geqslant 1$, that was established in [1] . In that paper, the authors used this fact to prove that the operator in (1.5) is similar to a contraction whenever it is polynomially bounded. Proposition 3.2 will play the same role in the proof of the corresponding result for weighted Bergman shifts. We should point out that one can deduce the factorization below from decomposition results in $[\mathbf{2 1}]$ and $[\mathbf{2 2}]$; however, we will provide an alternative proof based on duality.

Proposition 3.2. Let $\alpha>-1$. Then $D A(\mathbb{D}) \odot L_{a}^{1}\left(\mu_{\alpha}\right)=L_{a}^{1}\left(\mu_{\alpha+1}\right)$ with comparable norms.

Proof. Let $f \in A(\mathbb{D}), g \in L_{a}^{1}\left(\mu_{\alpha}\right)$. First we notice that $f g \in L_{a}^{1}\left(\mu_{\alpha}\right)$ and, therefore, $(f g)^{\prime} \in D L_{a}^{1}\left(\mu_{\alpha}\right)=L_{a}^{1}\left(\mu_{\alpha+1}\right)$. Moreover,

$$
\left\|(f g)^{\prime}\right\|_{L_{a}^{1}\left(\mu_{\alpha+1}\right)} \sim\left\|(f g)^{\prime}\right\|_{D L_{a}^{1}\left(\mu_{\alpha}\right)} \leqslant\|f g\|_{L_{a}^{1}\left(\mu_{\alpha}\right)} \leqslant\|f\|_{\infty}\|g\|_{L_{a}^{1}\left(\mu_{\alpha}\right)} .
$$

On the other hand, $g \in L_{a}^{1}\left(\mu_{\alpha}\right)$ and so $g^{\prime} \in D L_{a}^{1}\left(\mu_{\alpha}\right)=L_{a}^{1}\left(\mu_{\alpha+1}\right)$. Thus, $f g^{\prime} \in L_{a}^{1}\left(\mu_{\alpha+1}\right)$ with

$$
\left\|f g^{\prime}\right\|_{L_{a}^{1}\left(\mu_{\alpha+1}\right)} \leqslant C\|f\|_{\infty}\|g\|_{L_{a}^{1}\left(\mu_{\alpha}\right)} .
$$

It follows, since $f^{\prime} g=(f g)^{\prime}-f g^{\prime}$, that

$$
D A(\mathbb{D}) \odot L_{a}^{1}\left(\mu_{\alpha}\right) \subseteq L_{a}^{1}\left(\mu_{\alpha+1}\right) .
$$

Having established the bounded inclusion

$$
j: D A(\mathbb{D}) \odot L_{a}^{1}\left(\mu_{\alpha}\right) \hookrightarrow L_{a}^{1}\left(\mu_{\alpha+1}\right),
$$

the proof of the proposition will be complete if we can show that $j$ is surjective or, equivalently, that its adjoint $j^{*}$ is bounded below. Identifying the dual of $L_{a}^{1}\left(\mu_{\alpha+1}\right)$ with BLOCH, it suffices to show that there is a constant $C>0$ such that

$$
\|b\|_{\text {BLOCH }} \leqslant C \sup \left\{\left|\int_{\mathbb{D}} \bar{b} f^{\prime} g \mathrm{~d} \mu_{\alpha+1}\right|:\|f\|_{\infty}=1,\|g\|_{L_{a}^{1}\left(\mu_{\alpha}\right)}=1\right\} .
$$


Suppose that the supremum above is finite for the function $b$ and denote this number by $M$. For $z \in \mathbb{D}$,

$$
b(z)=\int_{\mathbb{D}} \frac{b(\lambda)}{(1-\bar{\lambda} z)^{\alpha+3}} \mathrm{~d} \mu_{\alpha+1}(\lambda) .
$$

Differentiating under the integral sign and multiplying through by $z$ gives

$$
z b^{\prime}(z)=(\alpha+3) \int_{\mathbb{D}} \frac{b(\lambda) z \bar{\lambda}}{(1-\bar{\lambda} z)^{\alpha+4}} \mathrm{~d} \mu_{\alpha+1}(\lambda)
$$

By the definition of $M$,

$$
\left|z b^{\prime}(z)\right| \leqslant \frac{1}{2}(\alpha+3) M\left\|\frac{1}{(1-\bar{z} \lambda)^{1 / 2}}\right\|_{\infty}\left\|\frac{\lambda}{(1-\bar{z} \lambda)^{\alpha+5 / 2}}\right\|_{L_{a}^{1}\left(\mu_{\alpha}\right)} .
$$

Since

$$
\left\|\frac{1}{(1-\bar{z} \lambda)^{1 / 2}}\right\|_{\infty} \sim\left(1-|z|^{2}\right)^{-1 / 2} \text { and }\left\|\frac{1}{(1-\bar{z} \lambda)^{\alpha+5 / 2}}\right\|_{L_{a}^{1}\left(\mu_{\alpha}\right)} \sim\left(1-|z|^{2}\right)^{-1 / 2}
$$

on $\mathbb{D}$, it follows that $\left|z b^{\prime}(z)\right| \leqslant C M\left(1-|z|^{2}\right)$ for all $z \in \mathbb{D}$. Now fix $z \in \mathbb{D}$ and set $\rho=\max \left\{\frac{1}{2},|z|\right\}$. Then there exists $z_{0} \in \mathbb{D}$ such that $\left|z_{0}\right|>\rho$ and $\left|b^{\prime}(z)\right| \leqslant\left|b^{\prime}\left(z_{0}\right)\right|$. Thus,

$$
\left|b^{\prime}(z)\right|<(1 / \rho)\left|z_{0} b^{\prime}\left(z_{0}\right)\right| \leqslant(1 / \rho) C M\left(1-\left|z_{0}\right|^{2}\right) \leqslant 2 C M\left(1-|z|^{2}\right)
$$

and the proof is complete.

Let $\alpha, \beta>-1$. A bounded bilinear form $B$ on $L_{a}^{2}\left(\mu_{\alpha}\right) \times L_{a}^{2}\left(\mu_{\beta}\right)$ is called a Hankel form if, for all polynomials $p$,

$$
B(p f, g)=B(f, p g), \quad \forall f \in L_{a}^{2}\left(\mu_{\alpha}\right), \quad \forall g \in L_{a}^{2}\left(\mu_{\beta}\right) .
$$

By the symbol of a Hankel form $B$ we mean the unique function $b \in L_{a}^{2}\left(\mu_{\beta}\right)$ satisfying $B(1, g)=\int_{\mathbb{D}} g \bar{b} \mathrm{~d} \mu_{\beta}$ for all $g \in L_{a}^{2}\left(\mu_{\beta}\right)$. In this case,

$$
B(p, g)=\int_{\mathbb{D}} \bar{b} p g \mathrm{~d} \mu_{\beta}, \quad \forall g \in L_{a}^{2}\left(\mu_{\beta}\right), \quad \forall \text { polynomials } p .
$$

We can identify the Hankel form $B$ with a bounded operator $H_{b}: L_{a}^{2}\left(\mu_{\alpha}\right) \rightarrow L_{a}^{2}\left(\mu_{\beta}\right)$ via $B(f, g)=\left\langle f, H_{b} \tilde{g}\right\rangle_{L_{\alpha}^{2}\left(\mu_{\beta}\right)}$, where $\tilde{g}(z)=\overline{g(\bar{z})}$. Using normalized monomials for orthonormal bases of $L_{a}^{2}\left(\mu_{\alpha}\right)$ and $L_{a}^{2}\left(\mu_{\beta}\right)$, the matrix representing the operator $H_{b}$ has the form $\left(\alpha_{i, j} a_{i+j}\right)$, where $b(z)=\sum_{n=0}^{\infty} a_{n} z^{n}$ and

$$
\begin{aligned}
\alpha_{i, j} & =\sqrt{\frac{(\beta+2)_{i}}{i !}} \sqrt{\frac{(\alpha+2)_{j}}{j !}} \frac{(i+j) !}{(\beta+2)_{i+j}} \\
& \sim \frac{(i+1)^{(\beta+1) / 2}(j+1)^{(\alpha+1) / 2}}{(i+j+1)^{\beta+1}}
\end{aligned}
$$


(cf. [22]). For this reason, the operator $H_{b}$ is called a weighted Hankel operator. Note that, by definition, $H_{b} M_{z}=M_{z}^{*} H_{b}$, where $M_{z}$ is the operator of multiplication by the coordinate function $z$ on the respective space. Thus, $H_{b}$ is densely defined on polynomials by $H_{b} p=p\left(M_{z}^{*}\right) b$.

Necessary and sufficient conditions on the symbol $b$ for the operator $H_{b}$ to extend to a bounded operator from $L_{a}^{2}\left(\mu_{\alpha}\right)$ into $L_{a}^{2}\left(\mu_{\beta}\right)$ can be found in several places including $[\mathbf{3}, \mathbf{5}, \mathbf{2 2}, \mathbf{2 6}, \mathbf{2 8}]$. For the sake of completeness we provide a proof of the following criterion. Note that, in the proposition below, there is no loss of generality in assuming that $\beta \geqslant \alpha>-1$, since $H_{b}^{*}: L_{a}^{2}\left(\mu_{\beta}\right) \rightarrow L_{a}^{2}\left(\mu_{\alpha}\right)$ is also a weighted Hankel operator.

Proposition 3.3. Let $\beta \geqslant \alpha>-1$. The operator $H_{b}$, densely defined on polynomials by $H_{b} p=p\left(M_{z}^{*}\right) b$, extends to a bounded operator from $L_{a}^{2}\left(\mu_{\alpha}\right)$ into $L_{a}^{2}\left(\mu_{\beta}\right)$ if and only if there is a constant $C>0$ such that $\left|b^{\prime}(z)\right|\left(1-|z|^{2}\right)^{1+(\beta-\alpha) / 2} \leqslant C$ for all $z \in \mathbb{D}$.

Proof. In terms of Hankel forms, the operator $H_{b}$ is bounded if and only if there is a constant $C>0$ such that for all polynomials $f$ and $g$,

$$
\left|\int_{\mathbb{D}} \bar{b} f g \mathrm{~d} \mu_{\beta}\right| \leqslant C\|f\|_{L_{a}^{2}\left(\mu_{\alpha}\right)}\|g\|_{L_{a}^{2}\left(\mu_{\beta}\right)} .
$$

By Proposition 3.1, $L_{a}^{2}\left(\mu_{\alpha}\right) \odot L_{a}^{2}\left(\mu_{\beta}\right)=L_{a}^{1}\left(\mu_{(\alpha+\beta) / 2}\right)$ and the norms are comparable. This implies that the inequality (3.6) is equivalent to the inequality

$$
\left|\int_{\mathbb{D}} \bar{b} h \mathrm{~d} \mu_{\beta}\right| \leqslant \tilde{C}\|h\|_{L_{a}^{1}\left(\mu_{(\alpha+\beta) / 2}\right)}, \quad \forall h \in L_{a}^{1}\left(\mu_{(\alpha+\beta) / 2}\right) .
$$

The result now follows from Lemma 2.5.

\section{The similarity problem for weighted Bergman shifts}

For clarity, we will write $T_{\alpha}$ for the operator $M_{z}$ defined on $L_{a}^{2}\left(\mu_{\alpha}\right), \alpha>-1$. Fix $\alpha, \beta>$ -1 and suppose that the densely defined weighted Hankel operator $H_{b} p=p\left(T_{\beta}^{*}\right) b$ extends to a bounded operator from $L_{a}^{2}\left(\mu_{\alpha}\right)$ into $L_{a}^{2}\left(\mu_{\beta}\right)$. We define a $2 \times 2$ operator matrix $R_{b}$, acting on the direct sum $L_{a}^{2}\left(\mu_{\alpha}\right) \oplus L_{a}^{2}\left(\mu_{\beta}\right)$, by

$$
R_{b}=\left(\begin{array}{cc}
T_{\beta}^{*} & H_{b} \\
0 & T_{\alpha}
\end{array}\right) .
$$

It is easy to see that, if $p$ is a polynomial, then

$$
p\left(R_{b}\right)=\left(\begin{array}{cc}
p\left(T_{\beta}^{*}\right) & H_{b} p^{\prime}\left(T_{\alpha}\right) \\
0 & p\left(T_{\alpha}\right)
\end{array}\right) .
$$

Let $\delta_{b}$ be the map defined on polynomials by $\delta_{b}(p)=H_{b} p^{\prime}\left(T_{\alpha}\right)$. Since both $T_{\alpha}$ and $T_{\beta}$ are contractions, von Neumann's inequality implies that the operator $R_{b}$ is polynomially 
bounded if and only if $\delta_{b}$ extends to a bounded map of the disk algebra, $A(\mathbb{D})$, into $\mathcal{L}\left(L_{a}^{2}\left(\mu_{\alpha}\right), L_{a}^{2}\left(\mu_{\beta}\right)\right)$; in other words, if and only if there is a constant $C>0$ such that

$$
\left|\left\langle\delta_{b}(p) f, g\right\rangle\right| \leqslant C\|p\|_{\infty}\|f\|_{L_{a}^{2}\left(\mu_{\alpha}\right)}\|g\|_{L_{a}^{2}\left(\mu_{\beta}\right)}
$$

for all polynomials $p$, and $f$ and $g$ in the appropriate weighted Bergman spaces.

We are now ready to state the main result of this section.

Theorem 4.1. Let $\beta, \alpha>-1$. The following are equivalent.

(a) The operator $R_{b}$ is polynomially bounded.

(b) $\left\|H_{b^{\prime}}: L_{a}^{2}\left(\mu_{\alpha}\right) \rightarrow L_{a}^{2}\left(\mu_{\beta}\right)\right\|<\infty$.

(c) $\left\|H_{b} D: L_{a}^{2}\left(\mu_{\alpha}\right) \rightarrow L_{a}^{2}\left(\mu_{\beta}\right)\right\|<\infty$.

(d) There exists an operator $X \in \mathcal{L}\left(L_{a}^{2}\left(\mu_{\alpha}\right), L_{a}^{2}\left(\mu_{\beta}\right)\right)$ such that $H_{b}=T_{\beta}^{*} X-X T_{\alpha}$.

(e) The operator $R_{b}$ is similar to

$$
\left(\begin{array}{cc}
T_{\beta}^{*} & 0 \\
0 & T_{\alpha}
\end{array}\right)
$$

Proof. We start by proving that (a), (b) and (c) are all equivalent. As shown above, $R_{b}$ is polynomially bounded if and only if $\delta_{b}(p)=H_{b} p^{\prime}\left(T_{\alpha}\right)$ satisfies the estimate in (4.2) for some constant $C>0$. Since the operator $T_{\beta}$ is just a multiplication by $z$ and $H_{b} T_{\alpha}=T_{\beta}^{*} H_{b}$, we have that

$$
\left\langle\delta_{b}(p) f, g\right\rangle_{L_{a}^{2}\left(\mu_{\beta}\right)}=\left\langle p^{\prime}\left(T_{\beta}^{*}\right) H_{b} f, g\right\rangle=\left\langle H_{b} f, \tilde{p}^{\prime}\left(T_{\beta}\right) g\right\rangle=\left\langle H_{b} f, \tilde{p}^{\prime} g\right\rangle .
$$

Also, when $f$ is a polynomial, $H_{b} f=f\left(T_{\beta}^{*}\right) b$. By identifying the weighted Hankel operator $\delta_{b}(p)$ with a Hankel form on $L_{a}^{2}\left(\mu_{\alpha}\right) \times L_{a}^{2}\left(\mu_{\beta}\right)$, it follows that the estimate in (4.2) is equivalent to

$$
\left|\int_{\mathbb{D}} \bar{b} p^{\prime} f g \mathrm{~d} \mu_{\beta}\right| \leqslant C\|p\|_{\infty}\|f\|_{L_{a}^{2}\left(\mu_{\alpha}\right)}\|g\|_{L_{a}^{2}\left(\mu_{\beta}\right)}
$$

for all polynomials $p, f$ and $g$.

By Propositions 3.1, 3.2 and 2.4, respectively, we have that

$$
\begin{aligned}
D A(\mathbb{D}) \odot\left(L_{a}^{2}\left(\mu_{\alpha}\right) \odot L_{a}^{2}\left(\mu_{\beta}\right)\right) & =D A(\mathbb{D}) \odot L_{a}^{1}\left(\mu_{(\alpha+\beta) / 2}\right) \\
& =L_{a}^{1}\left(\mu_{((\alpha+\beta) / 2)+1}\right) \\
& =D L_{a}^{1}\left(\mu_{(\alpha+\beta) / 2}\right)
\end{aligned}
$$

and the norms are all comparable. Therefore, inequality (4.4) is equivalent to

$$
\left|\int_{\mathbb{D}} \bar{b} h^{\prime} \mathrm{d} \mu_{\beta}\right| \leqslant \tilde{C}\|h\|_{L_{a}^{1}\left(\mu_{(\alpha+\beta) / 2}\right)}, \quad \forall h .
$$


By Lemma 2.6, the inequality in (4.5) is equivalent to

$$
\left|\int_{\mathbb{D}} \overline{b^{\prime}} h \mathrm{~d} \mu_{\beta}\right| \leqslant C_{1}\|h\|_{L_{a}^{1}\left(\mu_{(\alpha+\beta) / 2}\right)}, \quad \forall h .
$$

Using Proposition 3.1 once again, we obtain that (4.6) is equivalent to

$$
\left|\int_{\mathbb{D}} \overline{b^{\prime}} f g \mathrm{~d} \mu_{\beta}\right| \leqslant \tilde{C}_{1}\|f\|_{L_{a}^{2}\left(\mu_{\alpha}\right)}\|g\|_{L_{a}^{2}\left(\mu_{\beta}\right)}, \quad \forall f, g .
$$

However, the inequality (4.7) is precisely the statement that the weighted Hankel operator $H_{b^{\prime}}$ extends to a bounded operator from $L_{a}^{2}\left(\mu_{\alpha}\right)$ into $L_{a}^{2}\left(\mu_{\beta}\right)$. Thus, conditions (a) and (b) are equivalent.

Alternatively, starting with (4.5) and using the factorizations

$$
D L_{a}^{1}\left(\mu_{(\alpha+\beta) / 2}\right)=L_{a}^{1}\left(\mu_{((\alpha+\beta) / 2)+1}\right)=L_{a}^{2}\left(\mu_{\alpha+2}\right) \odot L_{a}^{2}\left(\mu_{\beta}\right)=D L_{a}^{2}\left(\mu_{\alpha}\right) \odot L_{a}^{2}\left(\mu_{\beta}\right),
$$

established by Propositions 2.4 and 3.1, we see that (4.5) is equivalent to the inequality,

$$
\left|\int_{\mathbb{D}} \bar{b} f^{\prime} g \mathrm{~d} \mu_{\beta}\right| \leqslant \tilde{C}_{1}\|f\|_{L_{a}^{2}\left(\mu_{\alpha}\right)}\|g\|_{L_{a}^{2}\left(\mu_{\beta}\right)}, \quad \forall f, g,
$$

and (4.8) is the statement that $H_{b} D$ is bounded as an operator from $L_{a}^{2}\left(\mu_{\alpha}\right)$ into $L_{a}^{2}\left(\mu_{\beta}\right)$. Therefore, conditions (b) and (c) are equivalent.

Next, we show the implication $(\mathrm{c}) \Rightarrow(\mathrm{d})$. In terms of operators the product rule for derivatives can be written as $D M_{z}=I+M_{z} D$. Multiplying on the left by $H_{b}$ yields $H_{b} D M_{z}=H_{b}+H_{b} M_{z} D$. Using the fact that $H_{b} M_{z}=M_{z}^{*} H_{b}$ we obtain that

$$
H_{b}=T_{\beta}^{*}\left(-H_{b} D\right)-\left(-H_{b} D\right) T_{\alpha} .
$$

Since $H_{b} D$ is bounded by (c) one can take $X=-H_{b} D$ in (d).

In order to establish that $(\mathrm{d}) \Rightarrow(\mathrm{e})$ it suffices to note that if $X$ is an operator satisfying (d), then

$$
\left(\begin{array}{cc}
1 & X \\
0 & 1
\end{array}\right)^{-1}\left(\begin{array}{cc}
T_{\beta}^{*} & H_{b} \\
0 & T_{\alpha}
\end{array}\right)\left(\begin{array}{cc}
1 & X \\
0 & 1
\end{array}\right)=\left(\begin{array}{cc}
1 & -X \\
0 & 1
\end{array}\right)\left(\begin{array}{cc}
T_{\beta}^{*} & H_{b} \\
0 & T_{\alpha}
\end{array}\right)\left(\begin{array}{cc}
1 & X \\
0 & 1
\end{array}\right)=\left(\begin{array}{cc}
T_{\beta}^{*} & 0 \\
0 & T_{\alpha}
\end{array}\right) .
$$

Finally, it is a well-known consequence of von Neumann's inequality that if an operator is similar to a contraction, then it must be polynomially bounded. Since both $T_{\alpha}$ and $T_{\beta}$ are contractions, this establishes the implication (e) $\Rightarrow(\mathrm{a})$ and the theorem is proved.

Remark 4.2. By Proposition 3.3, the operator $H_{b^{\prime}}$ extends to a bounded operator from $L_{a}^{2}\left(\mu_{\alpha}\right)$ into $L_{a}^{2}\left(\mu_{\beta}\right), \beta \geqslant \alpha$, if and only if

$$
\sup _{z \in \mathbb{D}}\left|b^{\prime \prime}(z)\right|\left(1-|z|^{2}\right)^{((\beta-\alpha) / 2)+1}<\infty .
$$

In particular, if $\alpha=\beta$, then $\left\|H_{b^{\prime}}: L_{a}^{2}\left(\mu_{\alpha}\right) \rightarrow L_{a}^{2}\left(\mu_{\alpha}\right)\right\|<\infty$ if and only if $b^{\prime}$ belongs to BLOCH. More generally, if $n$ is a non-negative integer, then $\| H_{b^{\prime}}: L_{a}^{2}\left(\mu_{\alpha}\right) \rightarrow$ 
$L_{a}^{2}\left(\mu_{\alpha+2 n}\right) \|<\infty$ if and only if $b^{(n+1)} \in \mathrm{BLOCH}$. Therefore, in the case that $\beta=\alpha+2 n$ for some integer $n \geqslant 0$ we can add the additional condition

$$
b^{(n+1)} \in \mathrm{BLOCH}
$$

to the five equivalent conditions in Theorem 4.1.

\section{The joint similarity problem for weighted Bergman shifts}

In this section we determine necessary and sufficient conditions for a pair of commuting operators of the form as in (4.1) to be jointly polynomially bounded and jointly completely polynomially bounded. We show that in this case the two notions coincide.

The proof of the joint similarity result, Corollary 5.5 below, is based on a first-order restriction result from [11]. We now provide the relevant details. Let $\gamma>0$ and define the kernel $k_{\gamma}$ on $\mathbb{D} \times \mathbb{D}$ by

$$
k_{\gamma}(\lambda, z)=\frac{1}{(1-\bar{z} \lambda)^{\gamma}}=\sum_{n=0}^{\infty} \frac{(\gamma)_{n}}{n !} \bar{z}^{n} \lambda^{n},
$$

where $(\gamma)_{n}$ is defined in (2.3). Let $H\left(k_{\gamma}\right)$ denote the Hilbert space of analytic functions on $\mathbb{D}$ with reproducing kernel $k_{\gamma}$. Note that for $\alpha>-1$, the kernel $K_{\alpha}$, defined in $(2.1)$, is equal to $k_{\alpha+2}$. Since kernels uniquely determine the Hilbert space structure of the space they generate, it follows that $L_{a}^{2}\left(\mu_{\alpha}\right)=H\left(k_{\alpha+2}\right)$ and the norms are the same.

It is well known that $M_{z}$ on $H\left(k_{\gamma}\right)$ is unitarily equivalent to a weighted shift on $\ell^{2}$ with weight sequence $w_{n}=\sqrt{(n+1) /(n+\gamma)}$. In particular, $\left\|M_{z}\right\| \leqslant 1$ if and only if $\gamma \geqslant 1$. Moreover, if $0<\gamma<1$, then $M_{z}$ on $H\left(k_{\gamma}\right)$ is not power bounded [24].

We may identify the Hilbert space tensor product $H\left(k_{\gamma}\right) \otimes_{2} H\left(k_{\gamma}\right)$ with the Hilbert space of holomorphic functions on $\mathbb{D} \times \mathbb{D}$ generated by the kernel

$$
\left(k_{\gamma} \otimes k_{\gamma}\right)(\lambda, z):=k_{\gamma}\left(\lambda_{1}, z_{1}\right) k_{\gamma}\left(\lambda_{2}, z_{2}\right)
$$

(cf. [23]). The identification is the usual one: $f \otimes g \mapsto h(z, w)=f(z) g(w)$. The restriction map $r(f)(z)=f(z, z)$ maps $H\left(k_{\gamma}\right) \otimes_{2} H\left(k_{\gamma}\right)$ onto the space $H\left(k_{2 \gamma}\right)$ and satisfies $r r^{*}=1$. There are several nice proofs of this result $($ cf. $[\mathbf{9}, \mathbf{2 3}])$. A proof of the restriction result for any pair of kernels on any domain in $\mathbb{C}^{d}$ can be found in the second paper.

Theorem 5.1 below is a higher-order version of this result. Before stating it we need to define the $n$ th-order restriction operators. For $n \geqslant 0$ let $J_{n, \gamma}$ denote the $n$ th-order partial differential operators (acting on holomorphic functions in two variables) by

$$
J_{n, \gamma}(f)=\sum_{j=0}^{n} \frac{(-1)^{n-j}\left(\begin{array}{c}
n \\
j
\end{array}\right)}{(\gamma)_{j}(\gamma)_{n-j}} \frac{\partial^{n} f}{\partial z^{j} \partial w^{n-j}} .
$$

The $n$ th-order restriction operator is defined by

$$
r_{n, \gamma}=r J_{n, \gamma},
$$

where $r$ is the aforementioned restriction map. 
For all $n \geqslant 0, r_{n, \gamma}$ maps $H\left(k_{\gamma}\right) \otimes_{2} H\left(k_{\gamma}\right)$ into holomorphic functions on $\mathbb{D}$. As stated earlier, the range of the map $r_{0, \gamma}(=r)$ is the space $H\left(k_{2 \gamma}\right)$. It was shown in [11] that the range of $r_{n, \gamma}$, for $n \geqslant 1$, is $H\left(k_{2 \gamma+2 n}\right)$ and $r_{n, \gamma} r_{n, \gamma}^{*}=\nu_{n, \gamma}^{2} I$, where $\nu_{n, \gamma} \geqslant 0$. Note that if $\alpha>-1$, then $\gamma=(\alpha / 2)+1>\frac{1}{2}$ and $H\left(k_{2 \gamma+2 n}\right)=L_{a}^{2}\left(\mu_{\alpha+2 n}\right)$ with isometric inclusions and, thus, the range of the map $r_{n, \gamma}$ is $L_{a}^{2}\left(\mu_{\alpha+2 n}\right)$. We record this result below for future reference. The proof will be omitted since it can be found in [11].

Theorem 5.1 (see [11]). Let $\alpha>-1$ and set $\gamma=(\alpha / 2)+1$. For $n \geqslant 0$, let $V_{D}^{n, \gamma}$ denote the closure in $H\left(k_{\gamma}\right) \otimes_{2} H\left(k_{\gamma}\right)$ of the ideal generated by the polynomial $(z-w)^{n}$. Set $\nu_{n, \gamma}=\left\|(z-w)^{n}\right\|_{H\left(k_{\gamma}\right) \otimes_{2} H\left(k_{\gamma}\right)}$. The map $\nu_{n, \gamma}^{-1} r_{n, \gamma}: H\left(k_{\gamma}\right) \otimes_{2} H\left(k_{\gamma}\right) \rightarrow L_{a}^{2}\left(\mu_{\alpha+2 n}\right)$ is a co-isometry with kernel $\left(V_{D}^{n, \gamma} \ominus V_{D}^{n+1, \gamma}\right)^{\perp}$.

Fix $\alpha>-1$ and set $\gamma=(\alpha / 2)+1$. Let $M_{z_{i}}$ denote multiplication by the $i$ th coordinate function $z_{i}, i=1,2$, on $H\left(k_{\gamma}\right) \otimes_{2} H\left(k_{\gamma}\right)$. Define $\delta^{\gamma}$ on polynomials in two variables by

$$
\delta^{\gamma}(p)=r_{1, \gamma} p\left(M_{z_{1}}, M_{z_{2}}\right) r_{0, \gamma}^{*} \in \mathcal{L}\left(L_{a}^{2}\left(\mu_{\alpha}\right), L_{a}^{2}\left(\mu_{\alpha+2}\right)\right) .
$$

If $\alpha \geqslant 0$, then $\gamma \geqslant 1$ and, thus, $M_{z}$ is contractive on $H\left(k_{\gamma}\right)$. In this case, $\left(M_{z_{1}}, M_{z_{2}}\right)$ is a pair of commuting contractions on $H\left(k_{\gamma}\right) \otimes_{2} H\left(k_{\gamma}\right)$ and so by Ando's dilation theorem [2], $\delta^{\gamma}$ extends to a completely bounded map of $A\left(\mathbb{D}^{2}\right)$ into $\mathcal{L}\left(L_{a}^{2}\left(\mu_{\alpha}\right), L_{a}^{2}\left(\mu_{\alpha+2}\right)\right)$ with

$$
\left\|\delta^{\gamma}\right\|_{c b} \leqslant\left\|r_{1, \gamma}\right\|\left\|r_{0, \gamma}\right\|=\|z-w\|_{H\left(k_{\gamma}\right) \otimes_{2} H\left(k_{\gamma}\right)}, \quad \forall \gamma=\frac{1}{2} \alpha+1 \geqslant 1 .
$$

As mentioned above, in the case in which $\frac{1}{2}<\gamma<1$ (i.e. $-1<\alpha<0$ ), $M_{z}$ is not power bounded on $H\left(k_{\gamma}\right)$ and, hence, the pair $\left(M_{z_{1}}, M_{z_{2}}\right)$ is not jointly polynomially bounded on $H\left(k_{\gamma}\right) \otimes_{2} H\left(k_{\gamma}\right)$. Nevertheless, it will follow from Proposition 2.1 and Theorem 5.1 that $\delta^{\gamma}$ is completely bounded on $A\left(\mathbb{D}^{2}\right)$.

Note that $r_{1, \gamma}=\gamma^{-1} r(\partial / \partial z-\partial / \partial w)$ and a calculation shows that for all holomorphic functions $f, g$,

$$
r_{1, \gamma}(f g)=r_{1, \gamma}(f) r(g)+r(f) r_{1, \gamma}(g) .
$$

If $p$ is a polynomial and $f \in L_{a}^{2}\left(\mu_{\alpha}\right)$, then we have that

$$
\delta^{\gamma}(p) f=r_{1, \gamma}\left(p r_{0, \gamma}^{*}(f)\right)=r_{1, \gamma}(p) r_{0, \gamma} r_{0, \gamma}^{*}(f)+r_{0, \gamma}(p) r_{1, \gamma}\left(r_{0, \gamma}^{*}(f)\right) .
$$

By Theorem 5.1, $r_{0, \gamma}^{*}$ maps $L_{a}^{2}\left(\mu_{\alpha}\right)$ into $\left(V_{D}^{1, \gamma}\right)^{\perp} \subseteq \operatorname{Ker}\left(r_{1, \gamma}\right)$ and $r_{0, \gamma} r_{0, \gamma}^{*}(f)=f$. It follows that, for all polynomials $p$,

$$
\delta^{\gamma}(p) f=r_{1, \gamma}(p) f, \quad \forall f \in L_{a}^{2}\left(\mu_{\alpha}\right) .
$$

In other words, $\delta^{\gamma}(p)=M_{r_{1, \gamma}(p)}$, where $M_{r_{1, \gamma}(p)}$ is the operator of pointwise multiplication by the polynomial $r_{1, \gamma}(p)$ acting from $L_{a}^{2}\left(\mu_{\alpha}\right)$ into $L_{a}^{2}\left(\mu_{\alpha+2}\right)$. Thus, in order to prove that $\delta^{\gamma}$ is completely bounded on $A\left(\mathbb{D}^{2}\right)$ it suffices to show that there is a constant $C>0$ such that, for all $n \geqslant 1$ and for all $n \times n$ matrices of polynomials $\left(p_{i j}\right)$,

$$
\left\|\left(M_{r_{1, \gamma}\left(p_{i j}\right)}\right)\right\|_{\ell_{n}^{2}\left(L_{a}^{2}\left(\mu_{\alpha}\right)\right) \rightarrow \ell_{n}^{2}\left(L_{a}^{2}\left(\mu_{\alpha+2}\right)\right)} \leqslant C\left\|\left(p_{i j}\right)\right\|_{M_{n}\left(A\left(\mathbb{D}^{2}\right)\right)} .
$$

The inequality above will follow easily from the base case $n=1$ established in the lemma below. 
Lemma 5.2. Let $\alpha>-1$ and $\gamma=\frac{1}{2} \alpha+1$. Then, for all polynomials $p$,

$$
\left\|M_{r_{1, \gamma}(p)}\right\|_{L_{a}^{2}\left(\mu_{\alpha}\right) \rightarrow L_{a}^{2}\left(\mu_{\alpha+2}\right)} \leqslant \frac{\sqrt{2}}{\gamma} \sqrt{\frac{\alpha+3}{\alpha+1}}\|p\|_{\infty} .
$$

Proof. Since $r_{1, \gamma}=\gamma^{-1}(\partial / \partial z-\partial / \partial w)=\gamma^{-1} r_{1,1}$, it follows by Proposition 2.1 and (5.4) that

$$
\begin{aligned}
\left\|M_{r_{1, \gamma}(p)}\right\|_{L_{a}^{2}\left(\mu_{\alpha}\right) \rightarrow L_{a}^{2}\left(\mu_{\alpha+2}\right)} & \leqslant \gamma^{-1} \sqrt{\frac{\alpha+3}{\alpha+1}}\left\|M_{r_{1,1}(p)}\right\|_{L_{a}^{2} \rightarrow L_{a}^{2}\left(\mu_{2}\right)} \\
& =\gamma^{-1} \sqrt{\frac{\alpha+3}{\alpha+1}}\left\|\delta^{1}(p)\right\| \\
& \leqslant \gamma^{-1} \sqrt{\frac{\alpha+3}{\alpha+1}}\|z-w\|_{H^{2}\left(\mathbb{D}^{2}\right)}\|p\|_{\infty} \\
& =\gamma^{-1} \sqrt{2} \sqrt{\frac{\alpha+3}{\alpha+1}}\|p\|_{\infty} .
\end{aligned}
$$

Now we can prove the general case.

Theorem 5.3. Let $\alpha>-1$ and set $\gamma=\frac{1}{2} \alpha+1$. The map $\delta^{\gamma}$, densely defined on polynomials by the formula in (5.3), extends to a completely bounded map of $A\left(\mathbb{D}^{2}\right)$ into $\mathcal{L}\left(L_{a}^{2}\left(\mu_{\alpha}\right), L_{a}^{2}\left(\mu_{\alpha+2}\right)\right)$ with $\left\|\delta^{\gamma}\right\|_{c b} \leqslant(\sqrt{2} / \gamma)((\alpha+3) /(\alpha+1))$.

Proof. By (5.5) it suffices to show that the inequality (5.6) holds with constant $C=(\sqrt{2} / \gamma)((\alpha+3) /(\alpha+1))$. Let $n \geqslant 1$ and let $\left(p_{i j}\right)$ be an $n \times n$ matrix of polynomials in two variables. Let $F=\left(f_{1}, f_{2}, \ldots, f_{n}\right) \in \ell_{n}^{2}\left(L_{a}^{2}\left(\mu_{\alpha}\right)\right)$ and $G=\left(g_{1}, g_{2}, \ldots, g_{n}\right) \in$ $\ell_{n}^{2}\left(L_{a}^{2}\left(\mu_{\alpha+2}\right)\right)$ with

$$
\|F\|_{\ell_{n}^{2}\left(L_{a}^{2}\left(\mu_{\alpha}\right)\right)}=\|G\|_{\ell_{n}^{2}\left(L_{a}^{2}\left(\mu_{\alpha+2}\right)\right)}=1 .
$$

We then have that

$$
\begin{aligned}
\left|\left\langle\left(M_{r_{1, \gamma}\left(p_{i j}\right)}\right) F, G\right\rangle_{L_{a}^{2}\left(\mu_{\alpha+2}\right)}\right| & =\left|\sum_{i, j} \int_{\mathbb{D}} r_{1, \gamma}\left(p_{i j}\right)(z) f_{j}(z) \overline{g_{i}(z)} \mathrm{d} \mu_{\alpha+2}(z)\right| \\
& =\left|\int_{\mathbb{D}}\left\langle\left(r_{1, \gamma}\left(p_{i j}(z)\right)\right) F(z), G(z)\right\rangle_{\ell_{n}^{2}} \mathrm{~d} \mu_{\alpha+2}(z)\right| \\
& \leqslant \int_{\mathbb{D}}\left\|\left(r_{1, \gamma}\left(p_{i j}\right)(z)\right)\right\|_{M_{n}}\|F(z)\|_{\ell_{n}^{2}}\|G(z)\|_{\ell_{n}^{2}} \mathrm{~d} \mu_{\alpha+2}(z) \\
& \leqslant(\alpha+3) \sup _{z \in \mathbb{D}}\left\{\left\|\left(r_{1, \gamma}\left(p_{i j}\right)(z)\right)\right\|_{M_{n}}\left(1-|z|^{2}\right)\right\} \\
& \times \int_{\mathbb{D}}\|F(z)\|_{\ell_{n}^{2}}\|G(z)\|_{\ell_{n}^{2}}\left(1-|z|^{2}\right)^{\alpha+1} \mathrm{~d} A(z) .
\end{aligned}
$$

Using Hölder's inequality together with (5.7), we see that

$$
\int_{\mathbb{D}}\|F(z)\|_{\ell_{n}^{2}}\|G(z)\|_{\ell_{n}^{2}}\left(1-|z|^{2}\right)^{\alpha+1} \mathrm{~d} A(z) \leqslant \frac{1}{(\alpha+1)^{1 / 2}(\alpha+3)^{1 / 2}} .
$$


Consequently,

$$
\left|\left\langle\left(M_{r_{1, \gamma}\left(p_{i j}\right)}\right) F, G\right\rangle_{L_{a}^{2}\left(\mu_{\alpha+2}\right)}\right| \leqslant \frac{\alpha+3}{(\alpha+1)^{1 / 2}(\alpha+3)^{1 / 2}} \sup _{z \in \mathbb{D}}\left\{\left\|\left(r_{1, \gamma}\left(p_{i j}\right)(z)\right)\right\|_{M_{n}}\left(1-|z|^{2}\right)\right\} .
$$

Now, for $z \in \mathbb{D}$,

$$
\begin{aligned}
\left\|\left(r_{1, \gamma}\left(p_{i j}\right)(z)\right)\right\|_{M_{n}} & =\sup _{a, b \in \ell_{n}^{2},\|a\|=\|b\|=1}\left|\sum_{i, j} a_{j} \overline{b_{i}} r_{1, \gamma}\left(p_{i j}\right)(z)\right| \\
& =\sup _{a, b \in \ell_{n}^{2},\|a\|=\|b\|=1}\left|r_{1, \gamma}\left(\sum_{i, j} a_{j} \overline{b_{i}} p_{i j}\right)(z)\right|,
\end{aligned}
$$

and thus, by Proposition 2.1 and Lemma 5.2,

$$
\begin{aligned}
\left\|\left(r_{1, \gamma}\left(p_{i j}\right)(z)\right)\right\|_{M_{n}}\left(1-|z|^{2}\right) & =\sup _{a, b \in \ell_{n}^{2},\|a\|=\|b\|=1}\left|r_{1, \gamma}\left(\sum_{i, j} a_{j} \overline{b_{i}} p_{i j}\right)(z)\right|\left(1-|z|^{2}\right) \\
& \leqslant \sup _{a, b \in \ell_{n}^{2},\|a\|=\|b\|=1}\left\|M_{r_{1, \gamma}\left(\sum_{i, j} a_{j} \overline{b_{i}} p_{i j}\right)}\right\|_{L_{a}^{2}\left(\mu_{\alpha}\right) \rightarrow L_{a}^{2}\left(\mu_{\alpha+2}\right)} \\
& \leqslant \frac{\sqrt{2}}{\gamma} \sqrt{\frac{\alpha+3}{\alpha+1}} \sup _{a, b \in \ell_{n}^{2},\|a\|=\|b\|=1}\left\|\sum_{i, j} a_{j} \overline{b_{i}} p_{i j}\right\|_{\infty} \\
& =\frac{\sqrt{2}}{\gamma} \sqrt{\frac{\alpha+3}{\alpha+1}}\left\|\left(p_{i j}\right)\right\|_{M_{n}\left(A\left(\mathbb{D}^{2}\right)\right)} .
\end{aligned}
$$

Therefore,

$$
\left\|\left(M_{r_{1, \gamma}\left(p_{i j}\right)}\right)\right\|_{\ell_{n}^{2}\left(L_{a}^{2}\left(\mu_{\alpha}\right)\right) \rightarrow \ell_{n}^{2}\left(L_{a}^{2}\left(\mu_{\alpha+3}\right)\right)} \leqslant \frac{\sqrt{2}}{\gamma}\left(\frac{\alpha+3}{\alpha+1}\right)\left\|\left(p_{i j}\right)\right\|_{M_{n}\left(A\left(\mathbb{D}^{2}\right)\right)} .
$$

Fix $\alpha, \beta>-1$. We now consider the pair of operators

$$
R_{b}=\left(\begin{array}{cc}
T_{\beta}^{*} & H_{b} \\
0 & T_{\alpha}
\end{array}\right), \quad R_{-b}=\left(\begin{array}{cc}
T_{\beta}^{*} & H_{-b} \\
0 & T_{\alpha}
\end{array}\right)=\left(\begin{array}{cc}
T_{\beta}^{*} & -H_{b} \\
0 & T_{\alpha}
\end{array}\right),
$$

acting on the direct sum $L_{a}^{2}\left(\mu_{\beta}\right) \oplus L_{a}^{2}\left(\mu_{\alpha}\right)$, where $H_{b}: L_{a}^{2}\left(\mu_{\alpha}\right) \rightarrow L_{a}^{2}\left(\mu_{\beta}\right)$ is a bounded weighted Hankel operator. Since $H_{b} T_{\alpha}=T_{\beta}^{*} H_{b}$, the operators $R_{b}$ and $R_{-b}$ commute. If $p$ is a polynomial in two variables, then a straightforward computation shows that

$$
p\left(R_{b}, R_{-b}\right)=\left(\begin{array}{cc}
p\left(T_{\beta}^{*}, T_{\beta}^{*}\right) & \Delta_{b}(p) \\
0 & p\left(T_{\alpha}, T_{\alpha}\right)
\end{array}\right)
$$

where

$$
\Delta_{b}(p)=H_{b}\left(\frac{\partial p}{\partial z}-\frac{\partial p}{\partial w}\right)\left(T_{\alpha}, T_{\alpha}\right)
$$


If $\left(p_{i j}\right)$ is an $n \times n$ matrix of polynomials in two variables, then, employing a change of basis - the so-called canonical shuffle - we obtain that

$$
\left\|\left(p_{i j}\left(R_{b}, R_{-b}\right)\right)\right\|=\left\|\left(\begin{array}{cc}
\left(p_{i j}\left(T_{\beta}^{*}, T_{\beta}^{*}\right)\right) & \left(\Delta_{b}\left(p_{i j}\right)\right) \\
0 & \left(p_{i j}\left(T_{\alpha}, T_{\alpha}\right)\right)
\end{array}\right)\right\| .
$$

Since $T_{\beta}$ is a contraction, it follows that the pair $\left(T_{\beta}^{*}, T_{\beta}^{*}\right)$ is jointly completely polynomially bounded with constant $C=1$. The same conclusion holds for the pair $\left(T_{\alpha}, T_{\alpha}\right)$. It follows that the pair $\left(R_{b}, R_{-b}\right)$ is jointly (completely) polynomially bounded if and only if the map $\Delta_{b}$ extends to a (completely) bounded map of $A\left(\mathbb{D}^{2}\right)$ into $\mathcal{L}\left(L_{a}^{2}\left(\mu_{\alpha}\right), L_{a}^{2}\left(\mu_{\beta}\right)\right)$.

We now realize $\Delta_{b}$ as a pushout of the completely bounded derivation $\delta^{\gamma}$, where $\gamma=$ $\frac{1}{2} \alpha+1$, and deduce from Theorem 5.3 that the map $\Delta_{b}$ is completely bounded on $A\left(\mathbb{D}^{2}\right)$ whenever it is bounded on $A\left(\mathbb{D}^{2}\right)$.

Theorem 5.4. Let $\beta, \alpha>-1$ and $\gamma=\frac{1}{2} \alpha+1$. The following are equivalent.

(1) $\Delta_{b}$ is bounded on $A\left(\mathbb{D}^{2}\right)$.

(2) $\Delta_{b}$ is completely bounded on $A\left(\mathbb{D}^{2}\right)$.

(3) $\left\|H_{b}: L_{a}^{2}\left(\mu_{\alpha+2}\right) \rightarrow L_{a}^{2}\left(\mu_{\beta}\right)\right\|<\infty$.

(4) There is a constant $C>0$ such that, for all polynomials $p$ in two variables,

$$
\left\|p\left(T_{\beta}^{*}, T_{\beta}^{*}\right) b\right\|_{L_{a}^{2}\left(\mu_{\beta}\right)} \leqslant C\left\|\left(M_{z_{1}}-M_{z_{2}}\right) p\right\|_{H\left(k_{\gamma}\right) \otimes_{2} H\left(k_{\gamma}\right)} .
$$

(5) There is a constant $C>0$ such that, for all polynomials $p$ in two variables,

$$
\left\|\left(\frac{\partial p}{\partial z}-\frac{\partial p}{\partial w}\right)\left(T_{\beta}^{*}, T_{\beta}^{*}\right) b\right\| \leqslant C\|p\|_{H\left(k_{\gamma}\right) \otimes_{2} H\left(k_{\gamma}\right)} .
$$

Furthermore, if (3) holds, then

$$
\left\|\Delta_{b}\right\|_{c b} \leqslant \frac{\sqrt{2}(\alpha+3)}{\alpha+1}\left\|H_{b}: L_{a}^{2}\left(\mu_{\alpha+2}\right) \rightarrow L_{a}^{2}\left(\mu_{\beta}\right)\right\| .
$$

Proof. First we show that (1) implies (3). If (1) holds, then, using (5.9), the operator $R_{b}$ is polynomially bounded. Thus, by Theorem 4.1, the operator $H_{b} D: L_{a}^{2}\left(\mu_{\alpha}\right) \rightarrow$ $L_{a}^{2}\left(\mu_{\beta}\right)$ is bounded. Condition (3) now follows by Proposition 2.4.

Suppose now that (3) holds. Then, by Theorem 5.3, the map $p \mapsto H_{b} \delta^{\gamma}(p)$ is completely bounded on $A\left(\mathbb{D}^{2}\right)$ with

$$
\left\|H_{b} \delta^{\gamma}(\cdot)\right\|_{c b} \leqslant\left\|H_{b}: L_{a}^{2}\left(\mu_{\alpha+2}\right) \rightarrow L_{a}^{2}\left(\mu_{\beta}\right)\right\|\left\|\delta^{\gamma}\right\|_{c b} .
$$

If $p$ is a polynomial in two variables, then, by (5.5),

$$
H_{b} \delta^{\gamma}(p)=H_{b} M_{r_{1, \gamma}}(p)=\gamma^{-1} H_{b}\left(\frac{\partial p}{\partial z}-\frac{\partial p}{\partial w}\right)\left(T_{\alpha}, T_{\alpha}\right)=\gamma^{-1} \Delta_{b}(p) .
$$


Therefore, condition (3) implies (2) and, moreover,

$$
\left\|\Delta_{b}\right\|_{c b} \leqslant \gamma\left\|H_{b}: L_{a}^{2}\left(\mu_{\alpha+2}\right) \rightarrow L_{a}^{2}\left(\mu_{\beta}\right)\right\|\left\|\delta^{\gamma}\right\|_{c b} .
$$

Since, obviously, (2) implies (1), we have shown that conditions (1), (2) and (3) are all equivalent. The last statement of the theorem now follows from Theorem 5.3 and the inequality above.

It remains to show that conditions (3), (4) and (5) are all equivalent. We first observe that, for a polynomial $p$,

$$
r_{1, \gamma}\left(M_{z_{1}}-M_{z_{2}}\right) p=2 \gamma^{-1} r(p) .
$$

If $f(z, w)=(z-w) p(z, w)$, then, from the formula above,

$$
H_{b} r_{1, \gamma} f=2 \gamma^{-1} H_{b} r(p)=2 \gamma^{-1} p\left(T_{\beta}^{*}, T_{\beta}^{*}\right) b .
$$

By Theorem 5.1, the orthogonal complement of the kernel of $r_{1, \gamma}$ is contained in $V_{D}^{1, \gamma}$ and functions of the form as $f$ are dense in $V_{D}^{1, \gamma}$. Consequently, the estimate (4) is equivalent to the condition that $\left\|H_{b} r_{1, \gamma}: H\left(k_{\gamma}\right) \otimes_{2} H\left(k_{\gamma}\right) \rightarrow L_{a}^{2}\left(\mu_{\beta}\right)\right\|<\infty$. On the other hand,

$$
H_{b} r_{1, \gamma}(p)=r_{1, \gamma}(p)\left(T_{\beta}^{*}\right) b=\gamma^{-1}\left(\frac{\partial p}{\partial z}-\frac{\partial p}{\partial w}\right)\left(T_{\beta}^{*}, T_{\beta}^{*}\right) b
$$

for all polynomials $p$. Thus, condition (5) is the statement that $\| H_{b} r_{1, \gamma}: H\left(k_{\gamma}\right) \otimes_{2}$ $H\left(k_{\gamma}\right) \rightarrow L_{a}^{2}\left(\mu_{\beta}\right) \|<\infty$. Therefore, (4) and (5) are equivalent. Finally, by Theorem 5.1, $r_{1, \gamma}: H\left(k_{\gamma}\right) \otimes_{2} H\left(k_{\gamma}\right) \rightarrow L_{a}^{2}\left(\mu_{\alpha+2}\right)$ is a multiple of a partial isometry with range $L_{a}^{2}\left(\mu_{\alpha+2}\right)$. Therefore, the boundedness of $H_{b} r_{1, \gamma}$ is equivalent to condition (3). The proof is now complete.

The following result is an easy consequence of Theorems 5.4 and 4.1.

Corollary 5.5. Let $\beta, \alpha>-1$ and let $R_{b}$ and $R_{-b}$ be the operators defined in (5.8). The following are equivalent.

(a) The operator $R_{b}$ is polynomially bounded.

(b) The pair $\left(R_{b}, R_{-b}\right)$ is jointly polynomially bounded.

(c) The pair $\left(R_{b}, R_{-b}\right)$ is jointly completely polynomially bounded.

(d) There is an invertible operator $S$, acting on $L_{a}^{2}\left(\mu_{\alpha}\right) \oplus L_{a}^{2}\left(\mu_{\beta}\right)$, such that $\left\|S^{-1} R_{b} S\right\| \leqslant$ 1 and $\left\|S^{-1} R_{-b} S\right\| \leqslant 1$.

(e) $\left\|H_{b} D: L_{a}^{2}\left(\mu_{\alpha}\right) \rightarrow L_{a}^{2}\left(\mu_{\beta}\right)\right\|<\infty$.

Proof. The implications (c) $\Rightarrow$ (b) and (b) $\Rightarrow$ (a) are obvious. The equivalence of conditions (c) and (d) follows from Theorem 1.1. The equivalence of conditions (a) and (e) follows from Theorem 4.1. It remains to show that $(\mathrm{e}) \Rightarrow(\mathrm{c})$. If (e) holds then by Proposition $2.4, H_{b}$ extends to a bounded operator on $L_{a}^{2}\left(\mu_{\alpha+2}\right)$. Thus, by Theorem $5.4, \Delta_{b}$ is completely bounded on $A\left(\mathbb{D}^{2}\right)$. It follows by $(5.11)$ that the pair $\left(R_{b}, R_{-b}\right)$ is jointly completely polynomially bounded. 
Next we consider the more general similarity problem involving pairs of the form

$$
R_{X_{1}}:=\left(\begin{array}{cc}
T_{\beta}^{*} & X_{1} \\
0 & T_{\alpha}
\end{array}\right), \quad R_{X_{2}}:=\left(\begin{array}{cc}
T_{\beta}^{*} & X_{2} \\
0 & T_{\alpha}
\end{array}\right),
$$

where $X_{1}, X_{2} \in \mathcal{L}\left(L_{a}^{2}\left(\mu_{\alpha}\right), L_{a}^{2}\left(\mu_{\beta}\right)\right)$. The operators $R_{X_{1}}$ and $R_{X_{2}}$ commute if and only if $\left(X_{1}-X_{2}\right) T_{\alpha}=T_{\beta}^{*}\left(X_{1}-X_{2}\right)$; in other words, if and only if $X_{1}-X_{2}=H_{b}$ is a weighted Hankel operator. Assuming commutativity, we define a map $\delta_{\left(X_{1}, X_{2}\right)}$ on polynomials in two variables by

$$
p\left(R_{X_{1}}, R_{X_{2}}\right)=\left(\begin{array}{cc}
p\left(T_{\beta}^{*}, T_{\beta}^{*}\right) & \delta_{\left(X_{1}, X_{2}\right)}(p) \\
0 & p\left(T_{\alpha}, T_{\alpha}\right)
\end{array}\right) .
$$

Since $p \mapsto p\left(R_{X_{1}}, R_{X_{2}}\right)$ is a homomorphism, it is not hard to see that

$$
\delta(p q)=p\left(T_{\beta}^{*}, T_{\beta}^{*}\right) \delta(q)+\delta(p) q\left(T_{\alpha}, T_{\alpha}\right), \quad \forall p, q .
$$

Such a map $\delta$ is called a derivation. The derivation property (5.15) implies that the map $\delta$ is completely determined by the operators $X_{i}=\delta\left(z_{i}\right), i=1,2$.

Notice that $T_{\beta}$ and $T_{\alpha}$ are both contractions, so the pair $\left(R_{X_{1}}, R_{X_{2}}\right)$ is jointly (completely) polynomially bounded if and only if the map $\delta_{\left(X_{1}, X_{2}\right)}$ extends to a (completely) bounded map from $A\left(\mathbb{D}^{2}\right)$ into $\mathcal{L}\left(L_{a}^{2}\left(\mu_{\alpha}\right), L_{a}^{2}\left(\mu_{\beta}\right)\right)$. The following result states that, when considering the joint (complete) polynomial boundedness of such a pair, one may restrict attention to commuting pairs of the form $\left(R_{H_{b}}, R_{0}\right)=\left(R_{b}, R_{0}\right)$ and $\left(R_{X_{2}}, R_{X_{2}}\right)$, the latter pair being a one-variable object.

Proposition 5.6. Suppose that $H_{b}=X_{1}-X_{2}$ is a weighted Hankel operator. If $\delta_{\left(X_{1}, X_{2}\right)}$ extends to a (completely) bounded operator on $A\left(\mathbb{D}^{2}\right)$, then the derivations $\delta_{\left(H_{b}, 0\right)}$ and $\delta_{\left(X_{2}, X_{2}\right)}$ are both (completely) bounded on $A\left(\mathbb{D}^{2}\right)$ and, furthermore, $\delta_{\left(X_{1}, X_{2}\right)}(f)=\delta_{\left(H_{b}, 0\right)}(f)+\delta_{\left(X_{2}, X_{2}\right)}(f)$ for all $f \in A\left(\mathbb{D}^{2}\right)$.

Proof. A routine computation show that, for all integers $i, j \geqslant 1$,

$$
\delta_{\left(X_{1}, X_{2}\right)}\left(z_{1}^{i} z_{2}^{j}\right)=\sum_{k=0}^{j-1} T_{\beta}^{* i+j-1-k} X_{2} T_{\alpha}^{k}+\sum_{k=0}^{i-1} T_{\beta}^{* k} X_{1} T_{\alpha}^{i+j-1-k} .
$$

Since $X_{1}=H_{b}+X_{2}$, it follows that

$$
\delta_{\left(X_{1}, X_{2}\right)}\left(z_{1}^{i} z_{2}^{j}\right)=\delta_{\left(H_{b}, 0\right)}\left(z_{1}^{i} z_{2}^{j}\right)+\delta_{\left(X_{2}, X_{2}\right)}\left(z_{1}^{i} z_{2}^{j}\right) .
$$

Thus, the additive property holds for all polynomials $p(z, w)$.

If $\delta_{\left(X_{1}, X_{2}\right)}$ extends to a (completely) bounded map on $A\left(\mathbb{D}^{2}\right)$, then the pair $\left(R_{X_{1}}, R_{X_{2}}\right)$ is jointly (completely) polynomially bounded and, in particular, the operator $R_{X_{2}}$ is (completely) polynomially bounded. It follows that pair $\left(R_{X_{2}}, R_{X_{2}}\right)$ is jointly (completely) polynomially bounded. But this is equivalent to the condition that $\delta_{\left(X_{2}, X_{2}\right)}$ is (completely) bounded on $A\left(\mathbb{D}^{2}\right)$. Since $\delta_{\left(H_{b}, 0\right)}=\delta_{\left(X_{1}, X_{2}\right)}-\delta_{\left(X_{2}, X_{2}\right)}$ on a dense subset of $A\left(\mathbb{D}^{2}\right)$, we conclude that $\delta_{\left(H_{b}, 0\right)}$ is necessarily (completely) bounded on $A\left(\mathbb{D}^{2}\right)$. 
Remark 5.7. The derivation $\delta_{\left(H_{b}, 0\right)}$ corresponds to the pair $\left(R_{b}, R_{0}\right)$. By the proposition above,

$$
2 \delta_{\left(H_{b}, 0\right)}=\delta_{\left(H_{b},-H_{b}\right)}+\delta_{\left(H_{b}, H_{b}\right)} .
$$

Corollary 5.5 asserts that $\delta_{\left(H_{b},-H_{b}\right)}$ is bounded on $A\left(\mathbb{D}^{2}\right)$ if and only if it is completely bounded on $A\left(\mathbb{D}^{2}\right)$ and, furthermore, the boundedness of the operator $H_{b} D$ is both a necessary and sufficient condition for the complete boundedness of $\delta_{\left(H_{b},-H_{b}\right)}$. Theorem 4.1 implies the same result for the derivation $\delta_{\left(H_{b}, H_{b}\right)}$ and, in particular, the derivation $\delta_{\left(H_{b}, H_{b}\right)}$ is (completely) bounded if and only if $\delta_{\left(H_{b},-H_{b}\right)}$ is (completely) bounded. It follows, by Proposition 5.6, that the derivation $\delta_{\left(H_{b}, 0\right)}$ is bounded on $A\left(\mathbb{D}^{2}\right)$ if and only if it is completely bounded on $A\left(\mathbb{D}^{2}\right)$, and this is the case if and only if the operator $H_{b} D$ is bounded. In other words, Corollary 5.5 holds for the pair $\left(R_{b}, R_{0}\right)$.

Corollary 5.8. Let $\left(R_{X_{1}}, R_{X_{2}}\right)$ be a pair of commuting operators of the form in (5.13) and suppose that the pair is jointly polynomially bounded. The following are equivalent.

(1) The operator $R_{X_{1}}$ is similar to a contraction.

(2) The pair $\left(R_{X_{1}}, R_{X_{2}}\right)$ is jointly similar to a pair of contractions.

(3) The operator $R_{X_{2}}$ is similar to a contraction.

Proof. If the pair $\left(R_{X_{1}}, R_{X_{2}}\right)$ is jointly polynomially bounded, then, by Proposition 5.6, the derivation $\delta_{\left(H_{b}, 0\right)}$ is bounded where $H_{b}=X_{1}-X_{2}$. By the remark above, this implies that $\delta_{\left(H_{b}, 0\right)}$ is completely bounded on $A\left(\mathbb{D}^{2}\right)$. Now, if (3) holds, then $\delta_{\left(X_{2}, X_{2}\right)}$ is completely bounded on $A\left(\mathbb{D}^{2}\right)$. Since $\delta_{\left(X_{1}, X_{2}\right)}=\delta_{\left(H_{b}, 0\right)}+\delta_{\left(X_{2}, X_{2}\right)}$ it follows that $\delta_{\left(X_{1}, X_{2}\right)}$ is completely bounded on $A\left(\mathbb{D}^{2}\right)$. Applying Theorem 1.1 we obtain that $(3) \Rightarrow(2)$. By considering $-H_{b}=X_{2}-X_{1}$, a similar argument shows that (1) $\Rightarrow(2)$. Since (2) implies both (1) and (3), the proof is complete.

Acknowledgements. S.H.F. was supported in part by NSF grant DMS-9996257. S.H.F. thanks R. Rochberg for clarifying the extent to which Corollary 5.5 holds in full generality (i.e. for all $\alpha>-1$ ) by improving the dilation result in [11].

\section{References}

1. A. B. Aleksandrov and V. V. Peller, Hankel operators and similarity to a contraction, Int. Math. Res. Not. no. 6 (1996), 263-275.

2. T. Ando, On a pair of commutative contractions, Acta Sci. Math. 24 (1963), 88-90.

3. J. Arazy, S. Fisher and J. Peetre, Hankel operators on weighted Bergman spaces, Am. J. Math. 110 (1988), 989-1054.

4. S. AXLER, The Bergman space, the Bloch space, and commutators of multiplication operators, Duke Math. J. 53 (1986), 315-332.

5. F. F. Bonsall, Hankel operators on the Bergman space for the disk, J. Lond. Math. Soc. 33 (1986), 355-364.

6. J. BouRGAin, On the similarity problem for polynomially bounded operators on Hilbert space, Israel J. Math. 54 (1986), 227-241.

7. J. F. Carlson, D. N. Clark, C. Foias, and J. P. Williams, Projective Hilbert $A(\mathbb{D})$ modules, New York J. Math. 1 (1994), 26-38. 
8. K. Davidson And V. I. Paulsen, Polynomially, bounded operators, J. Reine Angew. Math. 487 (1997), 153-170.

9. R. G. Douglas And V. I. Paulsen, Hilbert modules over function algebras, Pitman Research Notes in Mathematics, no. 217 (Longman Scientific and Technical, Harlow, 1989).

10. S. Ferguson, Ext groups and polynomially bounded operators, Proc. Am. Math. Soc. 124 (1996), 2779-2785.

11. S. H. Ferguson And R. Rochberg, Higher-order Hilbert-Schmidt Hankel forms on the ball, preprint.

12. C. Foias And J. P. Williams, On a class of polynomially bounded operators, preprint (1974).

13. S. JAnSOn, Hankel operators between weighted Bergman spaces, Ark. Math. 26 (1988), 205-219.

14. S. Janson And J. PeEtre, A new generalization of Hankel operators (the case of higher weights), Math. Nachr. 132 (1987), 313-328.

15. V. PAulsen, Completely bounded homomorphisms of operator algebras, Proc. Am. Math. Soc. 92 (1984), 225-228.

16. V. PAULSEN, Every completely polynomially bounded operator is similar to a contraction, J. Funct. Analysis 55 (1984), 1-17.

17. J. Peetre And R. RochberG, Higher order Hankel forms, in Multivariable operator theory, Seattle, WA, 1993, pp. 283-306, Contemporary Mathematics, vol. 185 (American Mathematical Society, Providence, RI, 1995).

18. V. PELleR, Estimates of functions of power bounded operators on Hilbert spaces, $J$. Operat. Theory 7 (1982), 341-372.

19. G. PISIER, A polynomially bounded operator on Hilbert space which is not similar to a contraction, J. Am. Math. Soc. 10 (1997), 351-369.

20. G. PISIER, Joint similarity problems and the generation of operator algebras with bounded length, Integ. Eqns Operat. Theory 31 (1998), 353-370.

21. R. Rochberg, Decomposition theorems for Bergman spaces and their applications, in Operators and function theory, Lancaster, 1984 (ed. S. Power), vol. 153, pp. 225-277 (Reidel, Dordrecht, 1985).

22. R. Rochberg And Z. Wu, A new characterization of Dirichlet type spaces and applications, Illinois J. Math. 37 (1993), 101-122.

23. N. SAlinas, Products of kernel functions and module tensor products, in Topics in operator theory, pp. 219-241, Operator Theory: Advanced Applications, vol. 32 (Birkhäuser, Basel, 1988).

24. A. SHIELDS, Weighted shift operators and analytic function theory, Topics in Operator Theory, Mathematical Surveys, vol. 13, pp. 49-128 (American Mathematical Society, Providence, RI, 1974).

25. D. Stengenga, Multipliers of the Dirichlet space, Illinois J. Math. 24 (1980), 113-139.

26. Z. Wu, Hankel and Toeplitz operators on Dirichlet spaces, Integ. Eqns Operat. Theory 15 (1992), 503-525.

27. Z. Wu, The predual and second predual of $W_{\alpha}$, J. Funct. Analysis 116 (1993), 314-334.

28. Z. Wu, A class of bilinear forms on Dirichlet type spaces, J. Lond. Math. Soc. 54 (1996), 498-514.

29. K. ZHU, Operator theory in function spaces, Monographs and Textbooks in Pure and Applied Mathematics, vol. 139 (Marcel Dekker, New York, 1990). 